\title{
Radio Frequency Heating of Ceramic Windows
} in Fusion Applications

John D. Fowler, Jr.

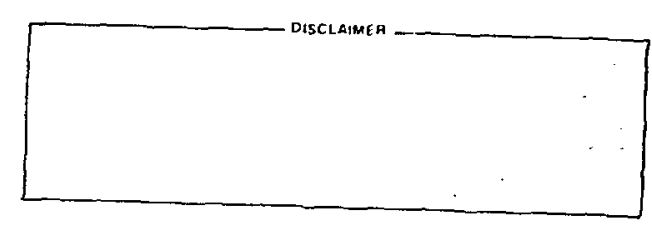


RADIO FREQUENCY HEATING OF CERAMIC WNDOWS IN FUSION APPLICATJONS

by

John D. Fowlet, Jr.

ABSTRACT

Ceramic windows will be used as material barriers for radio frequency plasma heating in fusion reactors. This report examines the theory behind $R$. F. heating phenomena. Heating calculations are presented for various wiriow materials, thicknesses, wavelengths, and power densities. The most pertinent material properties are loss tangent, thermal conductivity, dielectric corstant, strength, and radiation resistance. It is not known how these properties will be affected by the uperat in: environment of neutrons and gamina rays, but there is some indication that it will be adverse. Calculations indicate that among candidate materials, beryllium oxide offers the most promise because of its large thermal conductivity and relatively low loss tangent and dielectric constant. On the other hand, beryllia is susceptible to neutron damage, and this may adversely affect its electrical properties. In ther promising candidate is sapphire, paricularly at lower cemperatures where the thermal conductivity is high. Fused silica sulfers from low thermal conductivity and largc positive temperature coefficient for loss tangent, but it may be useful under some conditions. In sumary, calculations of heating can lead to elimination of some candidate materials and selection of others for further study.

\section{INTRODUCTION}

Heating of ceramics by penetrating electromagnetic radiation has been a topic of experimental study for at least 20 years. In one early study, various ceramic and glass windows were exposed to power densities of $300 \mathrm{MW} / \mathrm{m}^{2}\left(30 \mathrm{~kW} / \mathrm{cm}^{2}\right)$ tt frequencies of between 7 and $10 \mathrm{GHz} .^{1}$ Some but not all of the windors failed due to thermal stress. 
These early studies were undertaken to develop klystron tubes for radar applications. More recently, national and international commitments to develop a working fusion power reactor have led to a revival of interest in this area. This article addresses a calculational aspect of heating of ceramics due to losses from R. F. fields.

Because of their low loss characteristics, mechanical strength, and relative ability to contain tritfum, ceramic windows are prime candidates for radio frequency (R. F.) windows in fusion devices. ${ }^{2}$

Neutral beam injection and $R$. F. heating are two methods being considered for heating the plasma in a magnetic fusion device. The latter has the abvantage of requiring smaller physical penetrations into the vessel wall. In addition, there exists a well-developed radar technology, much of which can be drawn upon for microwave heating sources. ${ }^{3}$ Table I lists the relevant characteristics of the heating modes and power densities to be expected in a typical fusion reactor design. 4,5

One crucial difference between past studies of ceramic windows and current requirements occurs in the operating environment. Windows in fusion reactors, even if shielded, will experience at least moderate exposure to high and molium energy neutrons and gamma rays. The effect of this environment on losses and heating of windows is unknown. Failure of a window in an operating fusion reacror would be a catastrophic event. For these reasons, losses in windows under nearoperating conditions shouis be assessed experimentally.

The next section contains the theory which provides the basis for later calculations. The theory comes mostly from textbook references.

\section{THEORY}

\section{A. Fundamentals}

Electromagnetic waves propagating through solids become attenuated by loss of a portion of the field energy to the material. In metals this at tenuation is drastic. Attenuation through a copper sheet $10^{-6} \mathrm{~m}$ thick can be greater than $10^{6}$ at $10^{8} \mathrm{~Hz} .^{13}$ This is due to the ability of free electrons near the surface

\footnotetext{
General information about losses in insulators can be found in Kingery et al. 6 and von Hippe1. ${ }^{7}$ For interactions between fields and dielectrics, Stratton, ${ }^{8}$ von Hippe1, 9 and Collin 10 are useful references. Solutinns to the heat flow equation were obtained from Carslaw and Jaeger. 11 The finite differencing scheme for heat flow comes from Dusinberre. 12 
TABLE I

R. F. RESONANCE HEATING MODES UNDER CONSIDERATION FOR FUSION REACTORS 4,5

$\begin{array}{rrrrr}\text { Mode } & \text { Frequency } & \text { Power } & \text { Duration } & \text { Function } \\ \text { Ion Cyclotron } & 50-100 \mathrm{MHz} & 55 \mathrm{Mh} & 2 \mathrm{~min} & \text { Bulk heating } \\ \text { Lower Hybrid } & 2 \mathrm{GHz} & 55 \mathrm{Mh} & 100 \mathrm{~s} & \text { Bulk heating } \\ \text { Electron Cyclotron } & 80-140 \mathrm{GHz} & 1 \mathrm{MW} & 6 \mathrm{~s} & \text { Stistup }\end{array}$

to move about in response to the field forces. Insulators, on the other hand, typically allow large field penetration with small losses. The amount of loss is controlled by such material characteristics as impurities. traps, and vibrational modes, and by the frequency and intensity of the field.

The standard way to measure power losses in insulators involves coating two parallel faces of the material with conducting electrodes. Then a voltage is applied across them in series with a current-measuring device. Knowing the voltage and che current, one can determine the loss in the insulator. (In reality, there are three electrodes, as shown in Fig. 1. The purpose of the concentric:

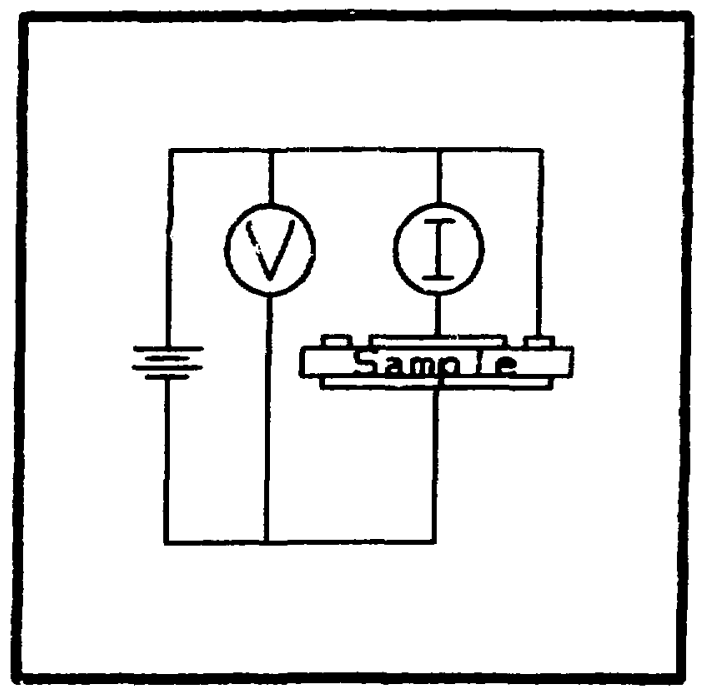

Fig. 1.

Connection of sample for measurment of D. C. conductivity. Center electrode, connected to current meter, is guarded by outer circular guard electrode. 
guard electrode is to prevent surface currents, which may be a large fraction of the total current in an insulator, from passing through the current meter.)

The insulator with its electrodes forms a parallel plate capacitor. In such a device the voltage across the terminals is proportional to the electrostatic charge stored, q, Eq. (1). The constant of proportionality is the capacitance, C.

$$
\mathrm{q}=\mathrm{CV} \text {. }
$$

The capacitance depends on the area of one of the plates, A, the separation, $d$, and the relative dielectric constant of the material, $k^{\top}$. The formula is

$$
C=\kappa^{\prime} \varepsilon_{0} \mathrm{~A} / \mathrm{d}=\kappa^{\prime} \mathrm{C}_{\mathrm{O}}
$$

where $\varepsilon_{0}$ is the permittivity of free space $\left[(36 \pi)^{-1} \times 10^{-9} \mathrm{farad} / \mathrm{m}\right]$ and $c_{0}$ is the capacitance of the electrodes in vacuum. Furthermore, $k^{\prime}$ equals $c^{\prime} / \ell_{0}$. where $\varepsilon^{\prime}$ is the permittivity of the material.

For an altemating voltage of frequency $f$, we have from Eq. (1) the following :

$$
q=C v_{0} \sin (\omega t) \text {, }
$$

where $V_{0}$ is the auplitude of the voltage and $\omega=2 \pi f$ is the angular frequency. In this case, the charge on the capacitor oscillates in phase with the applied voltage.

To derive an expression for the current entering or leaving a capacitor, use is made of the relation

$$
I=d q / d t=\omega C V_{0} \cos (\omega t)
$$

Comparing this with Eq. (3) it can be seen that the current and voltage both oscillate with the same frequency but $[$ remembering that $\sin i x)=\cos (x-90)]$, they are $90^{\circ}$ out of phase. The mathematical multiplier $j=\sqrt{-1}$ corresponds to a rotation of $90^{\circ}$ in the complex plane, so Eq. (4) can be rewritten as 


$$
I=\omega C j V \text { or } V=I(1 / j \omega C)=I(-j / \omega C) \text {. }
$$

The term in parentheses is called the capacitive reactance, and it is also the impedance, $z$, of this circuit.

It should be noted that there is no loss in a "pure" capacitor since the power loss equals the integral over a complete cyle of the voltage times the current. Thus

$$
\begin{aligned}
& P=1 / 2 \pi:{ }_{0}^{2 T} V_{0} \sin (\omega t) C V_{0} \cos (\omega t) d z= \\
& \omega C V_{0}^{2} / 4 \pi t_{0}^{2 \pi} \sin (2 \omega t) d t=\mathrm{CV}_{0}^{2} /\left.8 \pi \cos (2 \omega t)\right|_{0} ^{2 \pi}=0 .
\end{aligned}
$$

Real capacitors, however, always have :ome losses. One way to simulate this is to consider a real capacitor to be cmposed of a "pure" capacilor connerted in paralled with a resistive loss path (Fig. 2).

This circuit behaves as a capacilor with the dielectric constant replaced by a complex constant,

$$
*^{*}=k^{\prime}-\mathrm{j} k^{\prime \prime}
$$

This ian be seen by calculating the impedance for this parallel circuit:

$$
Z_{p}=k X_{r} /\left(R+X_{c}\right)=(-j k / \omega C) /(R-j / \omega C)=-j /(\omega C-j / R)
$$

The impedance for the "pure" capacitor with complex dielectric constant is

$$
Z_{c}=-j i \omega C=-j / \omega C_{0}\left(k^{\prime}-j k^{\prime \prime}\right)
$$

Equations (7) and (8) are identj.cal if

$$
\omega C_{0} K^{\prime \prime}=1 / R
$$

Remembering that 


$$
\sigma=\mathrm{d} / \mathrm{AR} \text {, }
$$

and using Eq. (2), Eq. (9) may be converted to

$$
\sigma=\omega \varepsilon^{\prime \prime} \text {. }
$$

Thus the conductivity is related to the imaginary coefficient of the dielectric constant.

Another useful quantity can be defined by considering the relation between the voltage and the current. From Ohm's Law and Eq. (8),

$$
I=V / z_{c}=V_{\omega} C_{0}\left(k^{\prime \prime}+j \kappa^{\prime}\right) .
$$

The current has two components: one which is in phase with the applied voltage, known as the loss current, and another which leads the voltage by $90^{\circ}$, the charging current (Fig. 3). The angle delta is defined as the angle by which the resultant current differs from $90^{\circ}$ with respect to the applies voltage and is a

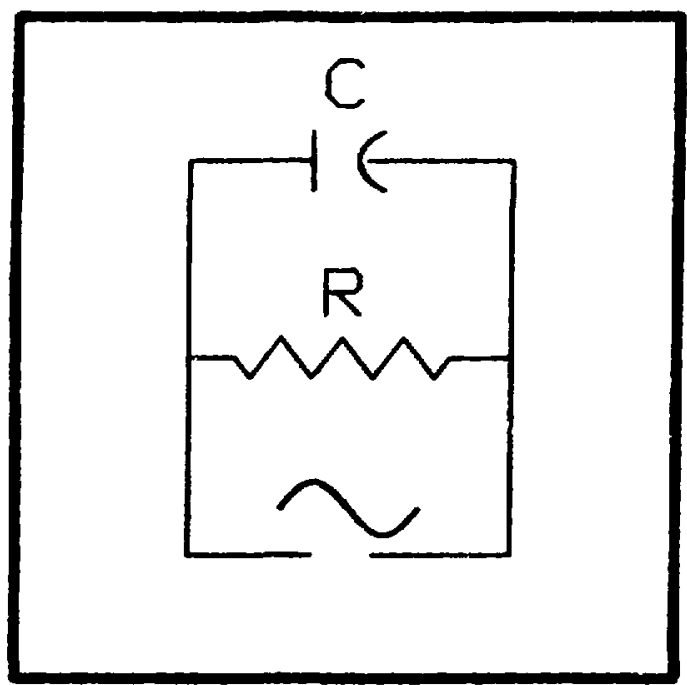

Fig. 2 .

Circuit diagram of a real capacitor, consisting of a pure capacitance connected in parallel with a resistive loss element.

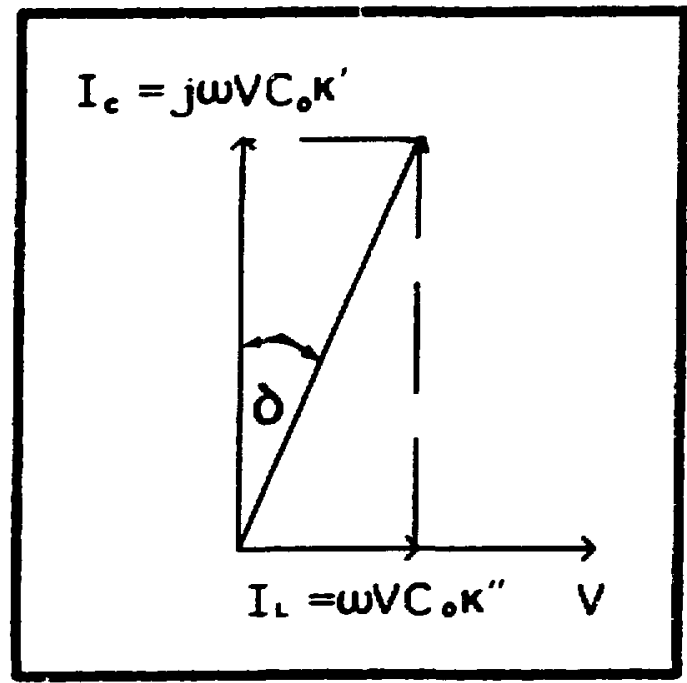

Fig. 3.

Voltage-current diagram showing composite current vector and loss angle. 
measure of the relative loss current. Delta is known as the loss angle and is related to the components of the dielectric periittivity by

$$
\tan \delta=c^{\prime \prime} / \varepsilon^{\prime}=\kappa^{\prime \prime} / k^{\prime} \text {. }
$$

Using Eq. (8),

$$
\sigma=\omega r^{\prime} \tan \delta
$$

This is the fundamental engineering equation that relates material properties (permittivity and loss angle) to electrical measurements (frequency and conductivity). It should be noted that for direct currents (that is, as $\omega \rightarrow 0$ ), insulator.j will still exhibit some finite corductivity or loss current. This implies [(Eq. (1I) and (14)] that as $\omega \rightarrow 0$, tan $\delta \rightarrow \infty$.

The quantity $\kappa^{\prime}$ tan $\delta$ is known as the relative loss factor. Figure 4 shows the frequency dependence of conductivity for varlous values of $\kappa^{\prime}$ tan $\delta$.

B. R. F. Fields

1. Attenuation Coefficient. Interactions between electromagnetic waves and matter are described by Maxwell's equations:

$$
\begin{aligned}
& \nabla \times \vec{E}=\partial \vec{B} / \partial t, \\
& \nabla \times \vec{H}=\partial \vec{D} / \partial t+\vec{J}, \\
& \nabla \cdot \vec{D}=0, \text { and } \\
& \nabla \cdot \vec{B}=0 .
\end{aligned}
$$

The relationship between the electric flux density $\vec{D}$ and the field strength $\vec{E}$ is given by

$$
\vec{D}=\varepsilon^{\prime} \vec{E}
$$

At this point it is convenient to describe the time variation of the fields by the complex exponential $\mathrm{e}^{\mathrm{j \omega t}}$. (This can be done with no loss of generality 


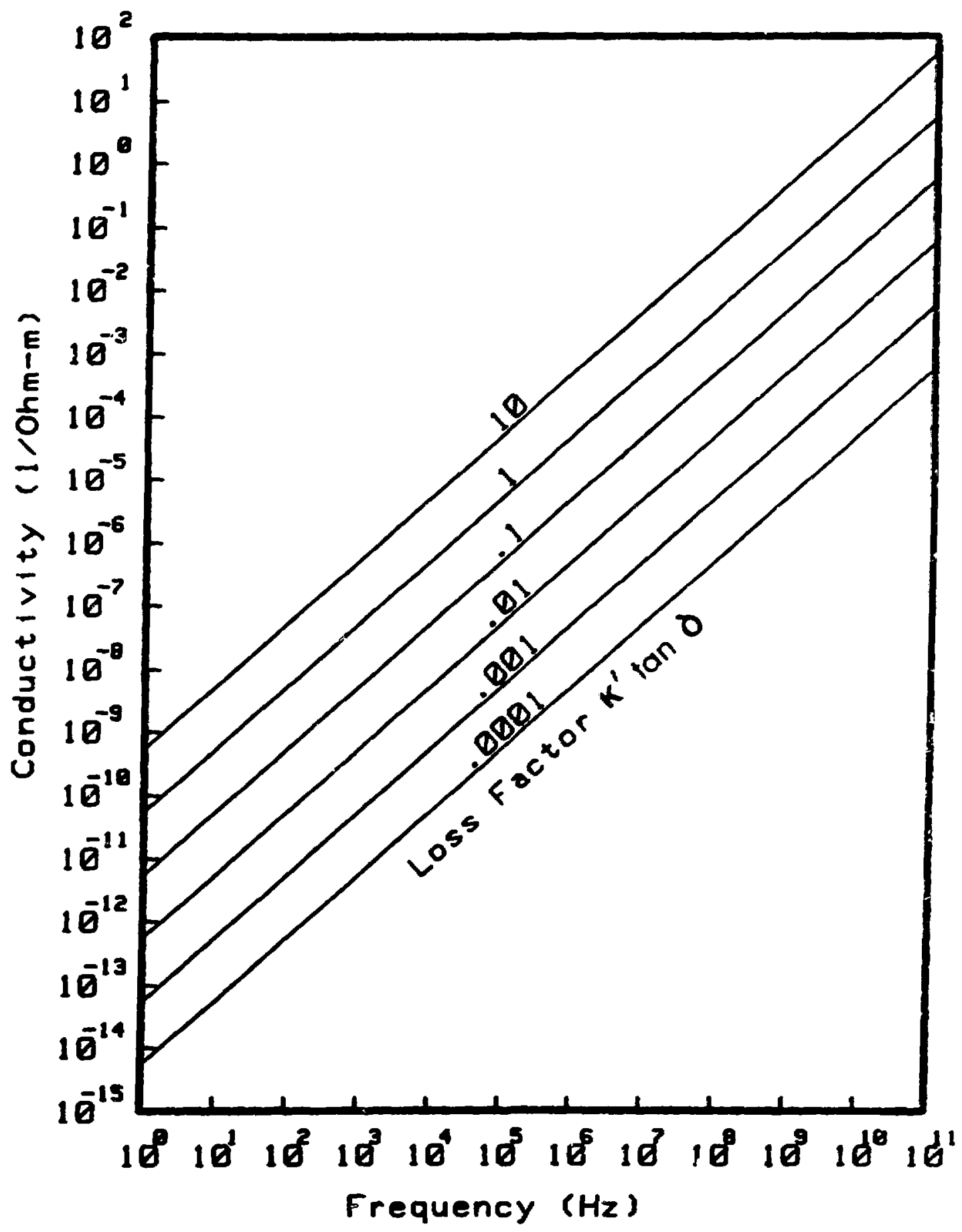

Fig. 4 .

Dependence of electrical conductivity on frequency and relative loss factor $\left(K^{\prime} \tan \delta\right)$. 
since any other time variation can be decomposed into a Fouries series of this type.) Then any partial derivative with respect to time becomes

$$
\partial \hat{F} /, t=j \omega r
$$

where if is an: ileld vector.

It can be seen from Eq. (15b) and Eq. (16) that

$$
\nabla \times \dot{H}=\varepsilon \dot{b} / 3 t+\vec{J} .
$$

For materials which obey Ohm's Law, the current density may be replaced by

$$
J=\omega \vec{E}=\omega \varepsilon^{\prime \prime} \vec{E}
$$

using Eq. (11). So Eq. (18) becomes

$$
\nabla \times \vec{H}=c \cdot \partial \vec{E} / \partial t+\omega E^{\prime \prime} \vec{E} .
$$

Using Eq. (17) on the last term of the above equation gives

$$
\nabla \times \vec{H}=\left(\varepsilon^{\prime}-j \varepsilon^{\prime \prime}\right) \partial \vec{E} / \partial t=\varepsilon * \partial \vec{E} / \partial t .
$$

Equation (15a) may be put into a form symmetrical to Eq. (21) by introduction of the complex permeability $\mu^{*}=\mu^{\prime}-j \mu^{\prime \prime}$, giving

$$
\nabla \times \vec{E}=-\mu^{*} \partial \vec{H} / \partial t .
$$

The next step is to separate the field vectors by taking the curl of Eq. (21) and Eq. (22), i.e.,

$$
\nabla \times \vee \times \vec{E}=-\mu^{*} \partial(\nabla \times \vec{H}) \partial t=-\mu^{*} E^{*} \partial^{2} \vec{E} / \partial t^{2},
$$

and similarly for $\vec{H}$. But, by the vector identity $\nabla \times \nabla \times \vec{A}=\nabla(\nabla \cdot A)-\nabla^{2} \vec{A}$ and using Eq. (15c) with zero charge density and Eq. (15d), the result is that

$$
\nabla \stackrel{2}{E}=\varepsilon^{*} \mu^{*} \partial^{2} \vec{E} / \partial t^{2}
$$


and

$$
\nabla^{2} \vec{H}=\epsilon^{*} \mu^{\star} \partial^{2} \vec{H} / \partial t^{2} .
$$

Notice that in separat Ing the field equations [for example, Eq. (23)], it wis assumed that $\mu^{*}$ and $\varepsilon^{*}$ are constant in space and ti-ile.

Equations (24) and (25) are examples of the wave equation and, rewritten it one space dinension, $x$, have solutions of the form

$$
\vec{E}=\vec{E}_{o} e^{j \omega t-\gamma x}
$$

and

$$
\vec{H}=\vec{H}_{o} e^{j \omega t-\gamma x} \text {. }
$$

These represent waves moving in the $x$-direction with a propagation faction i. which may be determined by substitution into $\mathrm{Eq}$. (24) or Bq. (25) (c) be

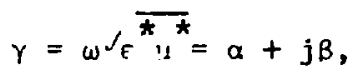

where $\alpha$ and $\beta$ are factors to be determined. Substitution for $\gamma$ into Eq. (26) and Eq. (27) gives

$$
\vec{E}=\vec{E}_{o} e^{-\alpha x_{e}} e^{-j(\omega t-\beta x)}
$$

and

$$
\vec{H}=\vec{H}_{0} e^{-\alpha x} e^{-j(\omega t-\beta x)} \text {. }
$$

The real term $\mathrm{e}^{-\alpha \mathrm{x}}$ represents a decaying exponential in space, where $\alpha$ is the attenuation factor. The term with $B$ represents a phase shift.

The waves have a spatial period given by 


$$
\lambda=2 \pi / B,
$$

where $\lambda$ is the wavelength in the material.

In general, $\alpha$ and $B$ will be functions of Iregucncy, pegmitcjuity, and permeability. For the determination of losices in R. $\therefore$ windows, we nay consider

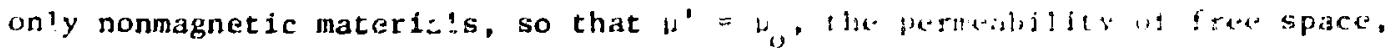
and $\mu^{\prime \prime}=0$. Then from Eq. (28),

$$
\alpha=\operatorname{Real} \text { part of }\left(j \omega\left[\mu_{0}\left\{\varepsilon^{\prime}-j \varepsilon^{\prime \prime}\right\}\right]^{1 / 2}\right)
$$

and

$$
B=\text { Imaginary part of }\left(j \omega\left[\mu_{0}\left\{\varepsilon^{\prime}-j \varepsilon^{\prime \prime}\right\}\right]^{1 / 2}\right) \text {. }
$$

Solving these gives

$$
\alpha=2 \pi / \lambda_{0}\left[k^{\prime} / 2\left\{\sqrt{\tan ^{2} \delta+1}-1\right\}\right]^{1 / 2}
$$

and

$$
B=2 \pi / \lambda_{0}\left[k^{\prime} / 2\left\{\sqrt{\tan ^{2} \delta+1}+1\right\}\right]^{1 / 2} \text {. }
$$

Use was made of the fact that the free-space wivelent's is given by

$$
\lambda_{0}=2 \pi / \omega\left(\varepsilon_{0} \mu_{0}\right)^{1 / 2}
$$

This can be seen from Eq. (28) and Eq. (31) with $x=0$.

We shall be primarily interested in the attesuation coefficient, $\alpha$. For small values of the loss angle, it is possible to approximate Eq. (34) by using

$$
\sqrt{\tan ^{2} \delta+1} \simeq 1+\frac{1}{2} \tan ^{2} \delta
$$

if $\tan \delta$ is small, so that 


$$
\alpha \simeq \pi / \lambda_{0} \sqrt{k^{r}} \tan \delta
$$

2. Relation between $\vec{E}$ and $\vec{H}$. To determine the relationship between the wave vectors $\vec{E}$ and $\vec{H}$ in the solutions, Eq. (26) and Eq. (27), it is necessary to return to Maxwell's Eq. (21) and Eq. (22). Since $\vec{E}$ and $\vec{H}$ depend only on time and the spatial dimension $x$,

$$
\partial \vec{E} / \partial y=\partial \vec{E} / \partial z=\partial \vec{H} / \partial y=\partial \vec{H} / \partial z=0
$$

Using this and writing out the components of Eq. (21) f.ives

$$
\begin{aligned}
\nabla \times \vec{H}= & \left|\begin{array}{ccc}
\vec{i} & \vec{j} & \vec{k} \\
\partial / \partial x & \partial / \partial y & \partial / \partial z \\
H & l & H
\end{array}\right|=-\vec{j} \partial H_{z} / \partial x+\vec{k} \partial H y / \partial x= \\
& \varepsilon^{*}\left(\vec{i} \partial E_{x} / \partial t+\vec{j}_{y} \partial E_{y} / \partial t+\vec{k} \partial E_{z} / \partial t\right),
\end{aligned}
$$

where $\vec{i}, \vec{j}$, and $\vec{k}$ are unit vectors in the $x, y$, and $z$ directions, respectively.

In like manner, from Eq. (22),

$$
-\vec{j} \partial E_{z} / \partial x+\vec{k} \partial E_{y} / \partial x=-\mu *\left(\vec{i} \partial H_{x} / \partial t+\vec{j} \partial H_{y} / \partial t+\vec{k} \partial H_{z} / \partial t\right)
$$

Equating components,

$$
\begin{array}{rlrl}
-\partial \mathrm{H}_{z} / \partial \mathrm{x} & =\varepsilon^{\star} \partial \mathrm{E}_{\mathrm{y}} / \partial \mathrm{t} & \partial \mathrm{E}_{\mathrm{z}} / \partial \mathrm{x} & =\mu{ }^{*} \partial \mathrm{H}_{\mathrm{y}} / \partial \mathrm{t} \\
\partial \mathrm{H}_{\mathrm{y}} / \partial \mathrm{x} & =\varepsilon^{\star} \partial \mathrm{E}_{\mathrm{z}} / \partial \mathrm{t} & -\partial \mathrm{E}_{\mathrm{y}} / \partial \mathrm{x} & =\mu{ }^{*} \partial \mathrm{H}_{\mathrm{z}} / \partial t \\
0 & =\varepsilon^{\star} \partial \mathrm{E}_{\mathrm{x}} / \partial \mathrm{t} & 0 & =\mu{ }^{*} \partial \mathrm{H}_{\mathrm{x}} / \partial \mathrm{t} .
\end{array}
$$

From the last two equations and from Eq. (15c) and Eq. (15d), it can be seen that the $x$-components of $\vec{E}$ and $\vec{H}$ are constant in space and time and might as well be set to zero. The $x$ - arid $y$-components are coupled, as can be seen by substitution of Eq. (26) and Eq. (27) into the above, for example, 


$$
\partial H_{y} / \partial x=-\gamma H_{y}=\epsilon^{*} \partial E_{z} / \partial t=\epsilon^{*} j \omega E_{z}
$$

The rat io

$$
|\vec{E}| /|\vec{H}|=\gamma / j \omega E^{*}=Z
$$

is the intrinsic impedance of the material. This maly be rewritten in several forms by the use of Eq. (28):

$$
Z=\gamma / j \omega \varepsilon^{*}=\sqrt{\mu^{*} / \varepsilon^{*}}=j \omega ; * \text { * } / \gamma \text {. }
$$

The intrinsic impedance of free space is

$$
z_{0}=\mu_{0} / \sqrt{\mu_{0} \varepsilon_{0}}=c \mu_{0}=377 \text { ohms, }
$$

where the speed of light is

$$
c=1 / \sqrt{\mu_{0} \varepsilon_{0}} \text {. }
$$

3. Conditions at Interfaces. When a wave encounters an abrupt change in the impedance of the propagating medium, part of the wave may be transmitted across the interface 1 part reflected back. The precise amounts of transmitted and reflected waves are determined by requiring that the tangential components of $\vec{E}$ and $\vec{H}$ be continuous across the interface.

We shall consider here only plane waves propagating perpendicularly to plane interfaces. In this case, for a single interface (Fig. 5), there are three pairs of $\vec{E}$ and $\vec{H}$ fields to consider: incident (i), transmitted ( $t$ ), and reflected ( $r$ ). The components of these fields may be written iy use of Eq. (26), (27), and (44a) as: 


$$
\begin{aligned}
& E_{i}=E_{0} e^{j \omega t-\gamma_{1} x}, H_{i}=E_{i} / Z_{1}, \\
& E_{r}=E_{1} e^{j \omega t+\gamma_{1} x}, H_{r}=-E_{r} / Z_{1}, \text { and } \\
& E_{t}=E_{2} e^{j \omega t-\gamma_{2} x}, H_{t}=E_{t} / z_{2} .
\end{aligned}
$$

Subscripts 1 and 2 refer to medium 1 and 2, respectively. The parameters $E_{1}$ and $\mathrm{E}_{2}$ are to be determined in tems of the incident wave amplitude $\mathrm{F}_{0}$. This is done by cquating components of $\vec{E}$. and $\overrightarrow{\mathrm{H}}$ across the interface:

$$
E_{0}+E_{1}=E_{2}
$$

ind

$$
E_{0}-E_{1}=Z_{1} E_{2} / Z_{2}
$$

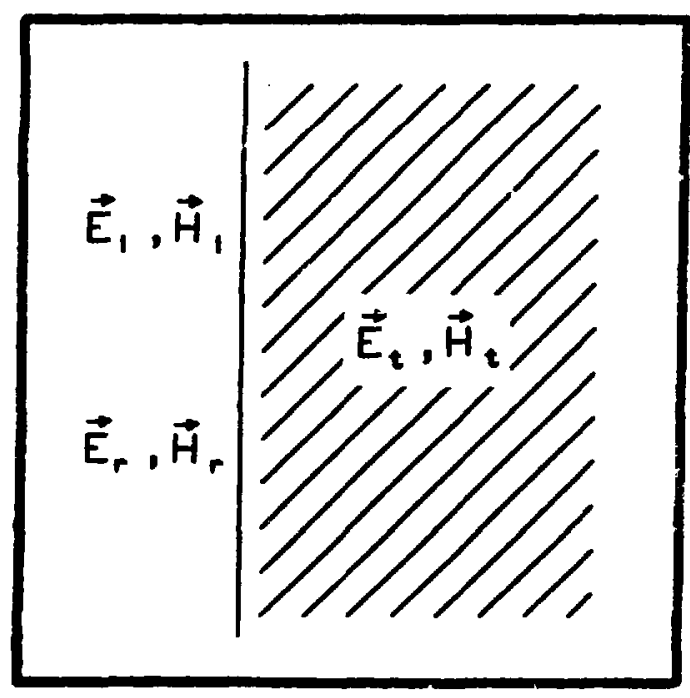

Fig. 5.

The semi-infinite slab with incident (i), reflectec $(r)$, and transmitted $(t)$ fields. 
These are readily solved to give

$$
E_{2}=\left[2 z_{2} /\left(z_{1}+z_{2}\right)\right] E_{o}
$$

and

$$
E_{1}=\left[\left(z_{2}-z_{1}\right) /\left(z_{1}+z_{2}\right)\right] E_{0}
$$

The time-averaged energy flow of the waves described by Eq. (47) is given by the real part of the Poynting vector:

$$
\operatorname{Re}(\vec{S})=\operatorname{Re}(\overrightarrow{\mathrm{E}} \times \overrightarrow{\mathrm{H}})=\frac{1}{2} \operatorname{Re}\left(\overrightarrow{\mathrm{E}} \times \overrightarrow{\mathrm{H}}^{* *}\right) \text {, }
$$

where the asterisk denotes compley conjugation. Le define the reflection and transmission coefficients as

$$
R=\left|\bar{s}_{r}\right| /\left|\bar{s}_{i}\right|=\left|E_{1}^{2} / E_{o}^{2}\right|=\left|\left[\left(z_{2}-z_{1}\right) /\left(z_{1}+z_{2}\right)\right]^{2}\right|
$$

and

$$
T=\left|\bar{s}_{t}\right| /\left|\bar{s}_{i}\right|=\left|\left(E_{2} / E_{o}\right)^{2} z_{1} / z_{2}\right|=\left|4 z_{1} z_{2} /\left(z_{1}+z_{2}\right)^{2}\right|,
$$

where the bars denote time averages. Thus the reflected and transmitced waves are completely described in terms of the impedance of the two media and the incident amplitude.

The conditions for a slab (two interfaces) are formally similar to those for a single interface, but the complex algebra involved is quite a bit more complicated. A computer program (Sec. IV) has been written to solve for the amplitudes and Poynting's vectors ir slab geometry.

In place of the three sets of fields shown in Fig. 5, the problem now has four field pairs (Fig. 6). These are written as

$$
E_{i}=E_{0} e^{j \omega t-\gamma_{1} x}, H_{i}=E_{i} / Z_{1},
$$




$$
\begin{aligned}
& E_{r}=E_{1} e^{j \omega t-\gamma_{1} x}, H_{r}=-E_{r} / Z_{1}, \\
& E_{m}=\left(E_{2}^{+} e^{-\gamma_{2} x}+E_{2}^{-} e^{\gamma_{2} x}\right) e^{j \omega t}, \\
& H_{m}=1 / Z_{2}\left(E_{2}^{+} e^{-\gamma_{2} x}-E_{2}^{-} e^{\gamma_{2} x}\right) e^{j \omega t}, \text { and } \\
& E_{t}=E_{3} e^{j \omega t-\gamma_{3} x}, H_{t}=E_{t} / Z_{3} .
\end{aligned}
$$

As in Eq. (48), a set of algrbraic equations in the amplituies and romplax impedances and propagation constants can be written by requiring continuity uf the fields at interfaces:

$$
\begin{aligned}
& E_{0}+E_{1}=E_{2}^{+}+E_{2}^{-}, \\
& E_{0}-E_{1}=z_{1} / z_{2}\left(E_{2}^{+}-\left(\overrightarrow{E_{2}}\right),\right.
\end{aligned}
$$

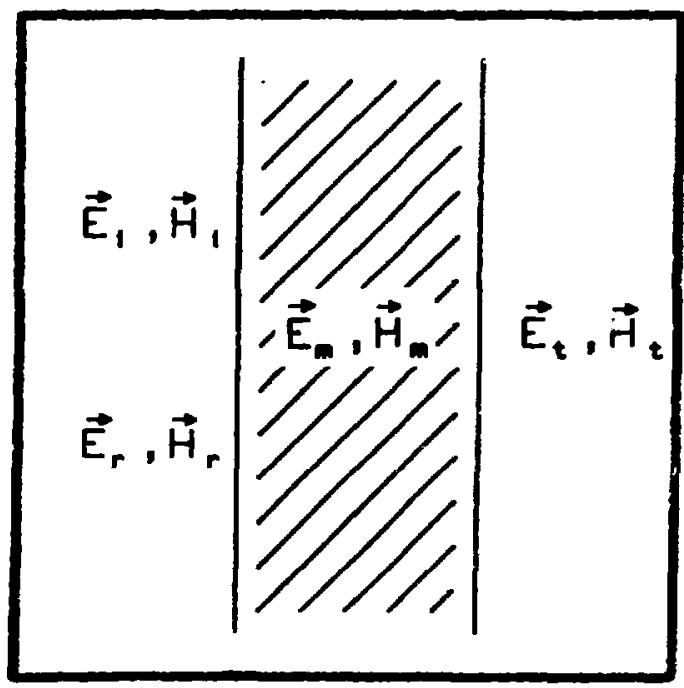

Fig. 6 .

The two-sided slab with incident (i), reflected $(r)$, transmitted $(t)$, and interior (m) fields. 


$$
\begin{aligned}
& E_{2}^{+} e^{-\gamma_{2} d}+E_{2}^{-e^{\gamma} 2^{d}}=E_{3} e^{-\gamma_{3} d} \text {, and } \\
& E_{2}^{+} e^{-\gamma_{2} d}-E_{2}^{-e^{\gamma}{ }^{d}}=E_{3} e^{-\gamma_{3} d_{2}}{ }_{2} / z_{3},
\end{aligned}
$$

where $d$ is the slab thickness.

The algebraic solution of Eq. (54) for the ratios of field intensities and for reflection and transmission coefficients and Poynting 's vector in the slab is difficult due to the complex nature of the quantities. Solutions can easily be programmed for a computer which has complex number routines. The slab problem will be discussed in conjunction with such a program in Sec. IV.

\section{Heat Conduction}

The next point for consideration is the flow of heat energy deposited by the R. F. fields disiussed above and the resulting temperature distributions in the material. A material with a large loss tangent may still be superior to one with small losses if its thermal and strength properties are better. It is a combination of loss, thermal conductivity, heat capacity, and strength which determines whether a window will hold or fail.

1. The Heat Flow Equation. The conduction of heat in solids is described by solutions to a partial differential equation which is of first order in the time derivative and norlinear and of second order in space:

$$
\rho c \partial T / \partial t=\partial / \partial x(K \partial T / \partial x)+\partial / \partial y(k \partial T / \partial y)+\partial / \partial x(k \partial T / \partial z)+A
$$

where $T=$ temperature, $\rho=$ density, $c=h e a t$ capacity, $K=$ thermal conductivity, and $A=$ source of heat. In general these are all functions of space and time, and the last four may depend on the temperature as well.

There are few known analytical solutions to Eq. (55). Usually solutions must be found by numerical, as opposed to analytical, methods; however, if the thermal properties can be regarded as constant in space and time, Eq. (55) simplifies to

$$
\nabla^{2} T-1 / K \partial T / \partial t=-A / K
$$


where $k=K / \rho c$ is the thermal diffusivity. This equation has several known useful solutions related to heat flow in windows.

(The limitation on thermal properties, i.e., constancy in space and time, is a particularly severe restriction on the themal conductivity, which usually decreases as temperature increases. This can lead to a self-reinforcing or ever. unstable situation, especlally if the loss tangent increases with temperature.)

We shall now consider solutions in one space dimension only: variations through the thickness of an infinite slab. This can be a useful approximation for R. F. windows if

(1) the window is thin with respect to its diameter, and

(2) cooling occurs through the faces and not through the edges.

Solutions exist for radial heat flow in cylindrical siabs, but they involve series of Bessel functions and their zeroes and solutions to transcendental equations. These must be determined numerically. A case can be made for solving the entire problem numerically from the outset.

2. Boundary Conditions. Solutions to Eq. (56) depend on the boundary conditions appropriate to the problem. There are three appropriate boundary conditions for problems considered here. Combinations of these conditions might also apply.

a. Constant Temperature. In this condition, the temperatures on bounding surfaces are specified as

$\mathrm{T}=$ const ant .

This condition simulates surfaces with high-capacity cooing. An example would be a fluid-cooled window, where the flid maintains a constant surface temperature on the window.

b. No Heat Flow. No heat is allowed to flow across the surface:

$$
\mathrm{dT} /\left.\mathrm{dx}\right|_{S}=0
$$

This condition simulates a thermally insulated window.

c. Radiative Heat Transfer. Heat flows between a body at temperature $T$ and its surroundings at $T_{o}$ by blackbody radiation: 


$$
\mathrm{d} T / \mathrm{dx}=\mathrm{\jmath} E\left(\mathrm{~T}^{4}-\mathrm{T}_{0}^{4}\right)
$$

where $E$ is the surface emissivity $(0<E<1)$ and $n$ is the StefanBoltzmann constant. Because of the small magnitude of $\sigma\left(0=1.37 \times 10^{-12} \mathrm{cal} /\right.$ $\mathrm{cm}^{2}$ sec $\mathrm{k}^{4}$ ), temperatures must be large or other modes of heat conduction must be very small before this method of heat tran fer becomes the major one. However, for sufficiently high temperature (because of the $\mathrm{T}^{4}$ dependence), radiative transfer is the dominant fcrm of iacot transfer.

There are very few known analytical solutions with this boundary condition. ivumerical methods must be used in most cases.

\section{Analytical Solutions. For certain boundary conditions there exist} analytical solutions for the heat-flow equation. Some of these will be listed and discussed below. They are usefu? as approximate solutions in some cases for the study of heating in windows. In addition, they were used to verify the accuracy of the finite difference model.

\section{a. Constant Initial and Surface Temperatures; Constant Heat}

Production $A_{0}-L \leq x \leq L$. This corresponds to boundary condition $(A)$ on both surfaces. The solution is

$$
\begin{aligned}
T= & T_{0}+A_{0} L^{2} / 2 K\left[1-(x / L)^{2}-32 / \pi^{3}\right. \\
& \left.\sum_{n=0}^{\infty}(-1)^{n} /(2 n+1)^{3} \cos (2 n+1) \pi x / 2 L e^{-k(2 n+1)^{2} \pi^{2} t / 4 L^{2}}\right] .
\end{aligned}
$$

This solution is typical of other solutions to the heat-flow equation in that the time dependence is expressed as an infinite series of decaying exponentials. In this instance, the strady-state portion is

$$
T_{s S}=T_{0}+A_{o} L^{2} / 2 K\left[1-(x / L)^{2}\right]
$$

This is parabolic in $x$ and reduces to the boundary condition $T=T_{0}$ at $x= \pm L$. The peak temperature is in the center $(x=0)$ and depends on the square of the thickness (thin windows run cooler!) and on the inverse of the thermal conductivity. 
The time required to reach steady state depends on the decaying exponential terms, which are functions of time, thermal diffusivity, and thickness. The bigger the thermal diffusivity and the thinner the window, the sooner it will reach equilibrium.

Because of the spatial symmetry of this problem, Eq. (60) is also the solution to the slab $0 \leq x \leq L$ with no heat flow across the surface $x=0$, i.e., bounary condition ( $B)$. In this case, however, for a slab of the same thickness as 'efore, the value of $L$ must be doubled. Since this is squared in the solution, Eq. (60), it is apparent that this window gets four times as hot as befire. b. Initial Temperature $\mathrm{T}$, Surface Temperature $T_{1}$; Heat Production at Constant Raté A, $0 \leq x \leq L$. A solution is

$$
\begin{aligned}
& T=T_{1}+A_{0} x(L-x) / 2 K-4 A_{0} L^{2} / K \pi^{3} \\
& \sum_{n=1}^{\infty}\left[e^{-(2 n-1)^{2} \pi^{2} k t / L^{2}} \text { sin }(2 n-1) \pi x / L /(2 n-1)^{3}\right] \\
& -4\left(T_{1}-T_{v}\right) / \pi \sum_{n=1}^{\infty}\left[e^{-(2 n-1)^{2} \pi^{2}+t / L^{2}} \sin (2 n-1) \pi x / 1 /(2 n-!)\right] .(62) \\
& \text { c. Pute of Heat Production a Function of Time. The solution for type }
\end{aligned}
$$

(A) boundary conditions is

$$
\begin{aligned}
T= & T_{0}+4 / \pi p c_{n=0}^{\infty}(-1)^{n} /(2 n+1) \cos (2 n+1) \pi x / 2 L \\
& \int_{0}^{t} A(\tau) e^{-\kappa(2 n+1)^{2} \pi^{2}(t-\tau) / 4 L^{2}} d \tau .
\end{aligned}
$$

There is no separate steady-state term here. This is to be expected as long as the heat source varies with time.

d. Rate of Heat Production a Function of Space. The solution for

type (A) boundary conditions is

$$
\begin{aligned}
T= & T_{0}+4 L / \pi^{2} K \sum_{n=1}^{\infty} 1 / n^{2}\left[1-e^{-k n^{2} \pi^{2} t / 4 L^{2}}\right] \cos n \pi x / 2 L \\
& \int_{-L}^{L} A\left(x^{\prime}\right) \cos n \pi x^{\prime} / 2 L d x^{\prime} .
\end{aligned}
$$


This does have a steady-state solution since the time-dependent terms eventually vanish.

Solutions (63) and (64) are useful if the loss to the window varies in time or space in a known manner.

4. Linerical Solutions. Often the problem at hand has no known analytical solution, or the nearest conditions for which an analytical solution exists do not offer a gand approximation to the real situation. Under these conditions, numerical (filite difference) methods may give more accurate results.

Most of the heat-flow calculations presented in Sec. IV were made using a program written in BASIC and run on a Hewlett-Packart HP9845B microcomputer.

The numerical method divides the materia: iniv a number of homogeneous cells or zones. The heat flow between a cell and its neighbors, plus any additional heat generated in the cell, is balanced with the temporal change of heac in the cell. Thus for cell (a),

$$
\sum_{n} K_{n a}\left(T n-T_{a}\right)+Q_{a}=C_{a} \Delta T_{a} / \Delta t
$$

where the sum is over all neighbors. "This is done over all cells; then the time step $\Delta t$ is incremented, and another cycle is calculated.

The handing of boundaries and criteria for the time step length are discussed elsewhere. 12

The accuracy of the finite difference method was assessed by comparing results with solutions to Eq. (60). Figures $7-10$ show results for three, six, nine, and twelve zones at short, medium, and long times. These calculations were for a two-wavelength-thick $\mathrm{Al}_{2} \mathrm{O}_{3}$ window at a frequency of $30 \mathrm{GHz}$. Heat capacity and thermal conductivity were constants. The loss tangent was 0.001 at an input power of $5 \mathrm{~kW} / \mathrm{cm}^{2}$. The boundary concitions were type (A) on one face and type (B) on the other.

The numbers plotted in Figs. 7-10 are centered on the zones and specify the number of zones in the calculation. The solid lines are the corresponding analytical solutions.

$* \overline{K_{n a}}$ is the thermal conductivity between $\operatorname{cell}(\mathrm{n})$ and cell (a), $\mathrm{C}_{\mathrm{a}}$ is the heat capacity of cell (a), and $Q_{a}$ is the heat source or sink (if anj) for cell (a). 

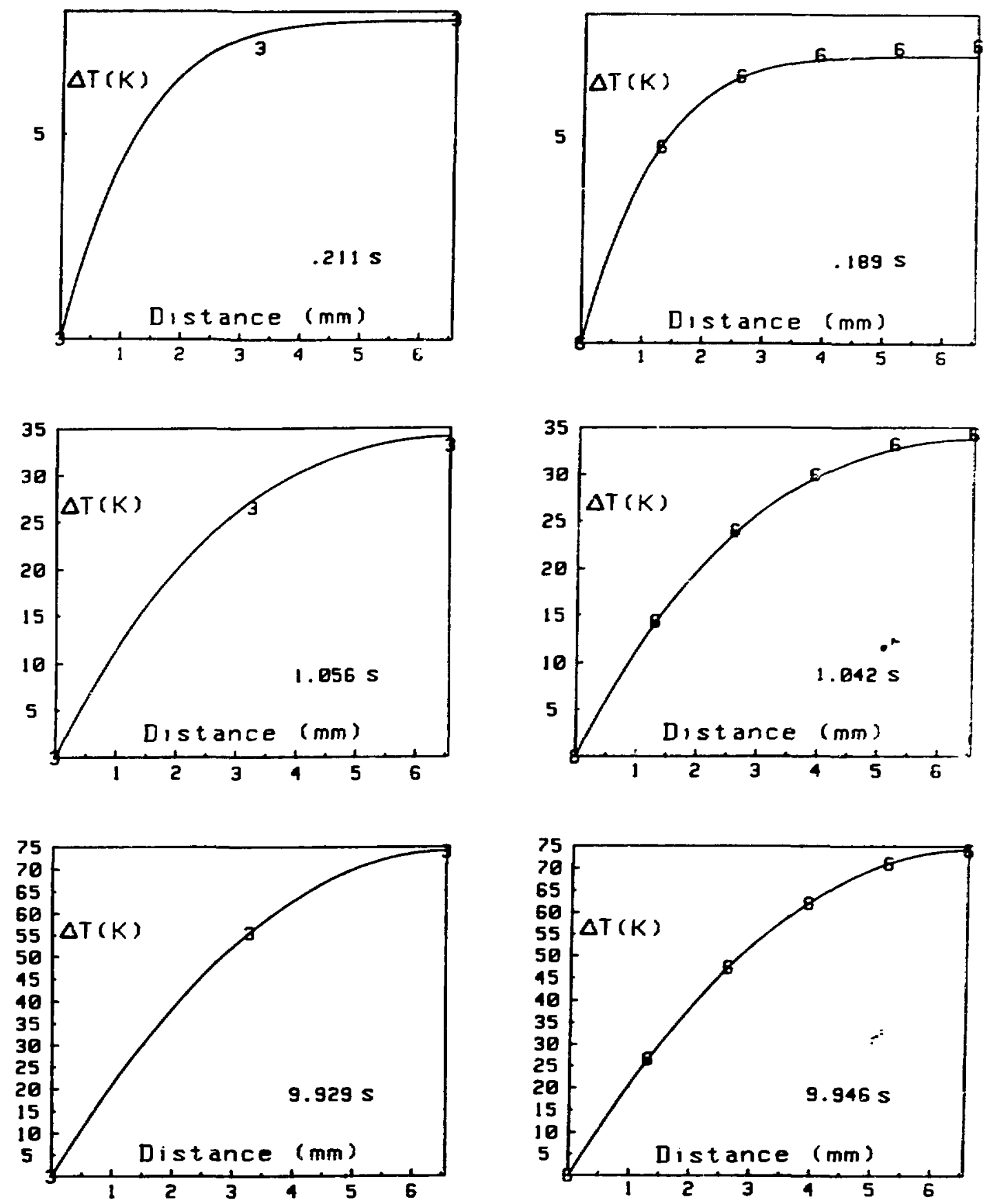

Fig. 7.

Numerical vs. analytical solution for face-cooled slab at various times Fig. 8.

Same as Fig. 7 but with 6 zones. using 3 zones. See text for conditions. 

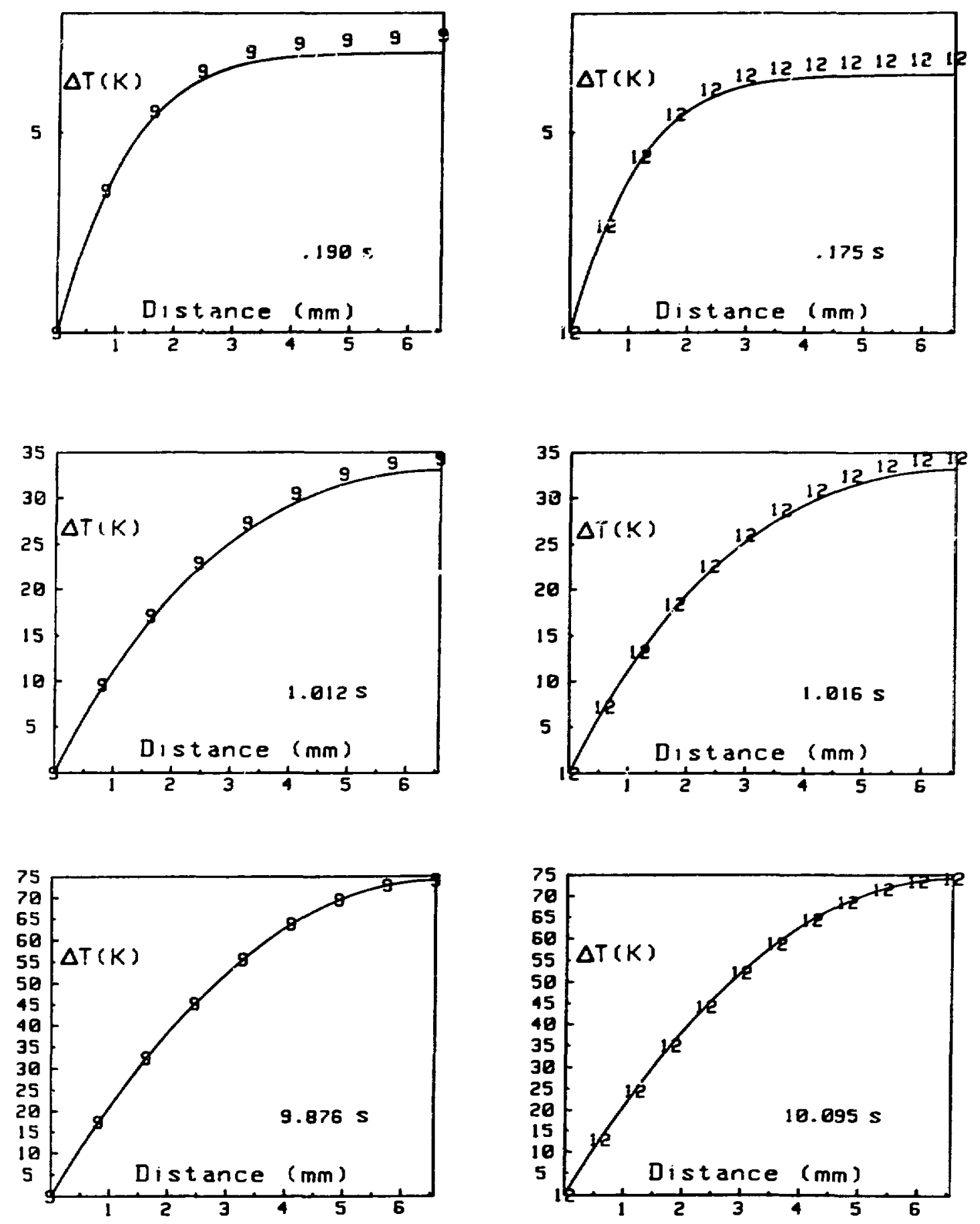

Fig. 9.

Same as Fig. 7 but with 9 zones.

Fig. 10.

Same as Fig. 7 but with 12 zones. 
It can be seen that even with three zones, the finite difference method adequately predicts the exact solution. The worst relative errors occur at small times in all the comparisons.

Based on these comparisons, it was decided to use six zones in the numerical calculations which appear in Sec. IV. The use of fewer than six zones would not define the curves well, and more zones would increase computer run time without increasing the accuracy of the results.

\section{DISCUSSION}

\section{A. Window Configurations}

Sizes, shapes, and precise locations of R. F. windows in fusion reactor designs have not been completely determined, but it is expected that dimensions will be on the order of millimeters to cerimeters in thickness and centimeters to tens of centimeters in diameter. Most windows which have been tested in microwave power systems $\mathrm{fall}$ in these ranges. $1,14,15$

Wirlow locations will be close enough to the plasma that significant ionizing and neutron irradiation will take place. Pressure gradients will be at least one atmosphere.

At the higher frequencies, the windows almost surely will be face cooled. A fluorocarbon, such as FC75, may be used. The loss in the coolant will undoubtedly be much greater than the loss in the window, requiring large coolant velocities and hence pressures, increasing the pressure gradient on the window. B. Losses

Loss tangents of candidate ceramics and glasses have bee.. found to be around $10^{-4}$ near room temperature for frequencies up to several $\mathrm{GHz}$. $7,16-18$ Recent results near $100 \mathrm{GHz}$ also indicate that loss tangents are in this range.

No data exist for losses in irradiated materials in the frequency range of interest, but data at $1 \mathrm{MHz}$ and below indicate that neutron 20,21 or concurrent ionizing 22 irradiation can increase loss tangents and conductive losses by a factor of 10 or more. If this occurs in the microwave range, it will cause considerable design problems. It is possible that minor impurities, especially those with large cross sections for neutrons, may be of crucial importance in a radiation environment. 


\section{CALCUILATIONS}

The principal factors affecting heat deposition from $R$. F. Fields are luss tangent, dielectric constant, and wavelength. The resulting temperature changes are decermined by thermal conductivity, heat capacity, and mode of couling.

\section{A. Losses Due to Currents}

For an insulator being heated by charge carriers (electrons, fons) mosins: under the influence of a potential $V$ across the thickness $d$. Eqs. (10) and (14) give the loss per unit area as

$$
P / A=V^{2} \omega E^{\prime}(\tan \delta) / d
$$

\section{B. Losses Due to Fields}

1. In an Infinite Medium. For an electromagnetic wave fropagating tnrough an insulating material, losses can be calculated from the fields, Fgs. (29) and (30) in the material. These depend on the attenuation coefficient $\alpha$ and the propagation constant $B$. The attenuation coefficient is sensitive to srall valuess of loss tangent, but $\beta$ is not. Typical values of $\alpha$ and $\beta$ are graphed in $F i g$. $! 1$.

The loss per unit area can be found using Eq. (50). The result is

$$
P / A P_{0}=1-e^{-2 \alpha x}
$$

A graph of this function appears in Fig. 12. It can be seen that for small values of the abscissa, the function is almost linear. In this zegion, the approximation Eq. (38) can be used to give

$$
\mathrm{P} / \mathrm{AP}{ }_{\mathrm{O}}=\left(2 \pi / \lambda_{0}\right) \sqrt{\mathrm{K}^{\prime}} \tan \delta \mathrm{x}
$$

2. Across an Interface. When an electromagnetic wave propagates across an interface, there is an immediate loss of field strength due to the change in impedance at the interface. Part of the energy is reflected back, leaving less to propagate across the boundary. The amounts reflected and transmitted can be found from Eqs. (51) and (52) and are primarily dependent on the value of the 
dielectric constant on each side of the interface. Figure 13 gives the reflection and transmission across a single interface from free space to material of dielectric constant $\kappa^{\prime}$.

3. In a Slab. For a slab, reflection can occur also at the rear interface, causing part of the waves to propagate back through the slab. This interfering wave can cancel or reinforce a portion of the original wave. The result is standing waves in the slab. The reflection, transmission, and loss coefficient: for a slab of $\mathrm{Al}_{2} \mathrm{O}_{3}$ are shown in Fig. 14. Noie that the loss coefficient is multiplied by a factor of 10 . It is apparent that at integral multiples of a halt wavelength in the slab, the reflection goes to zero and transmission reaches a maximum. These are the desirable conditions for iransmitting $R$. F. power through a window: that the thickness be a multiple of a half wavelength in the material.

Figures 15 and 16 show transmission, reflection, and loss multiplied by 10 for beryllium oxide and fused silica under the same conditions. It can be scen that the maxima of reflection and minima of loss are less extreme with lower dielectric constant.

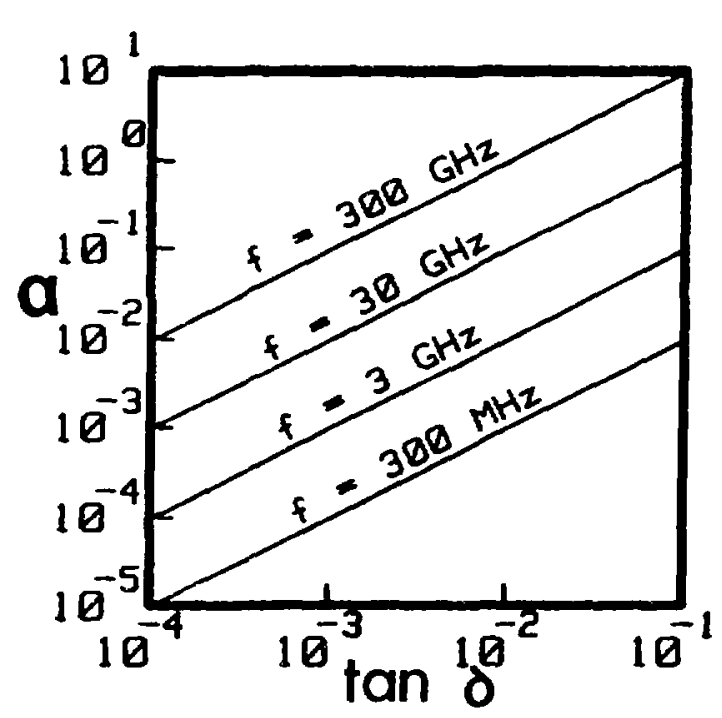

(a)

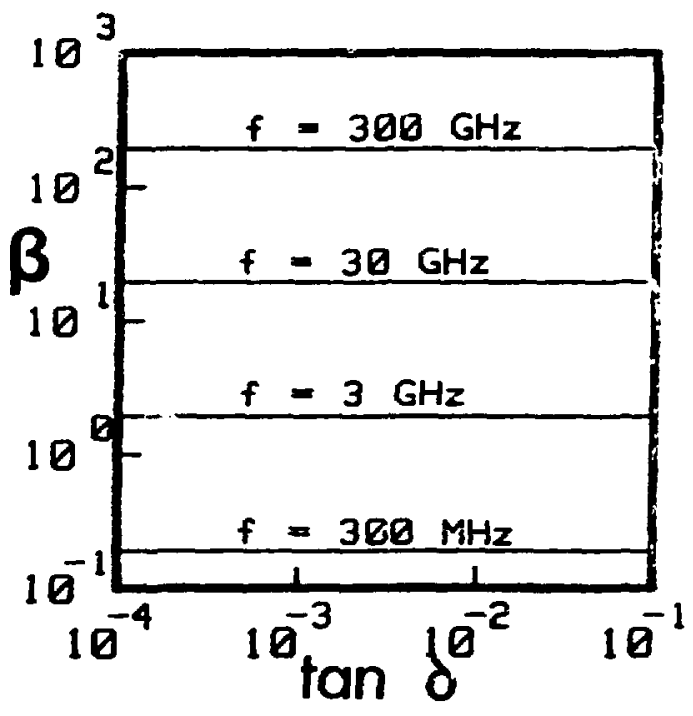

(b)

Fig. 11 .

(a) Attenuation coefficient $\alpha$ vs tan $\delta$ for various wavelengths. Note strong dependence on tan $\delta$. (b) Propagation constant $B$. Note lack of strong deperdence on $\tan \delta . k^{\prime}=9.3$ (alumina). 


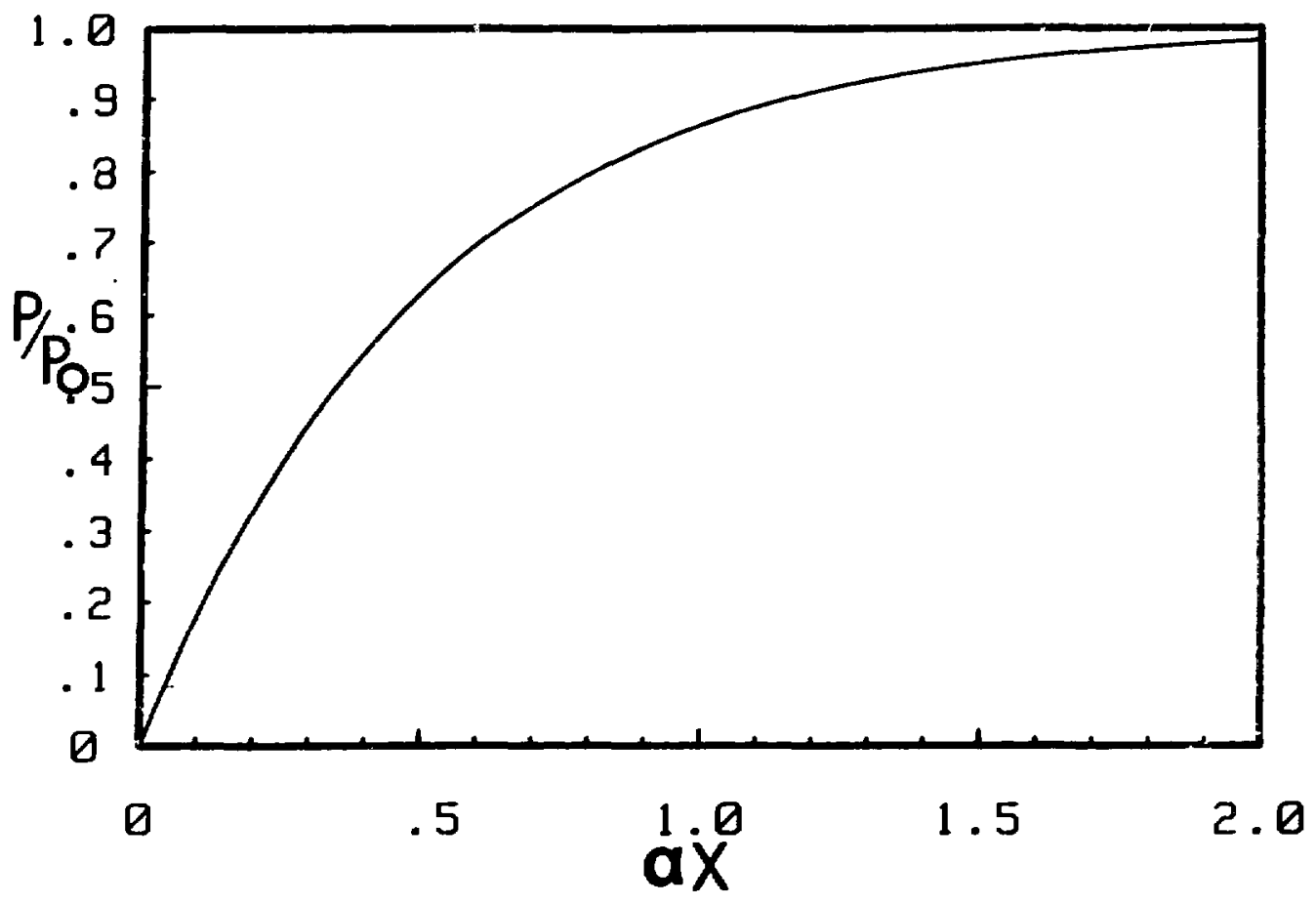

Fig. 12.

Plot of relative power loss per unit area as a function of normalized depth in an infinite dielectric medium.

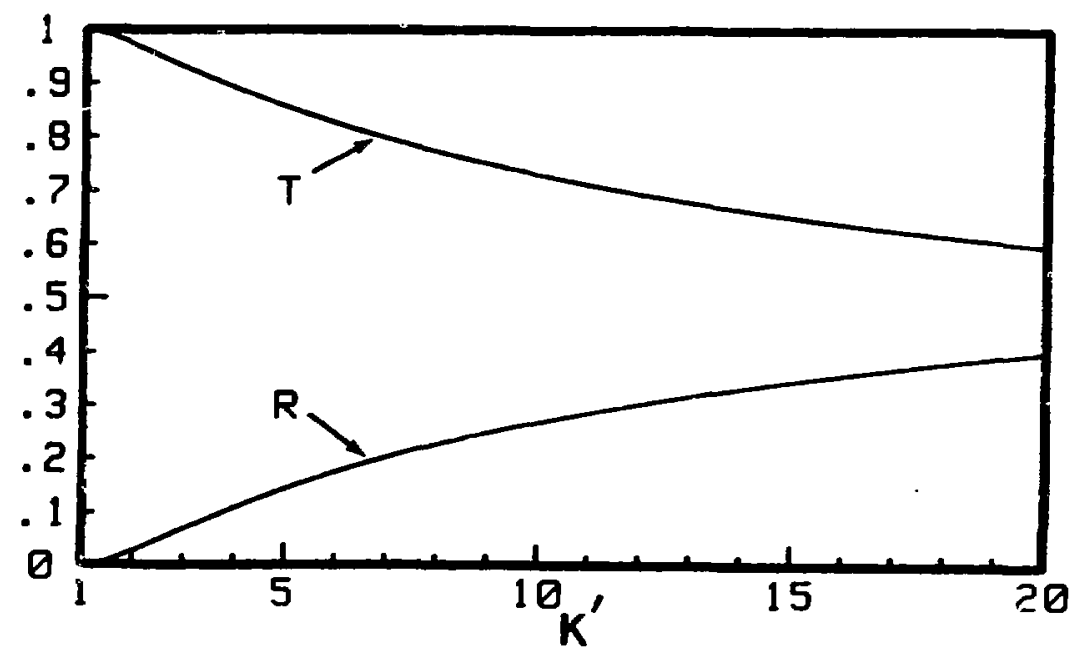

Fig. 13.

Reflection and transmission coefficient

for wave crossing from free space into medium of dielectric constant $\mathrm{K}^{\prime}$. 


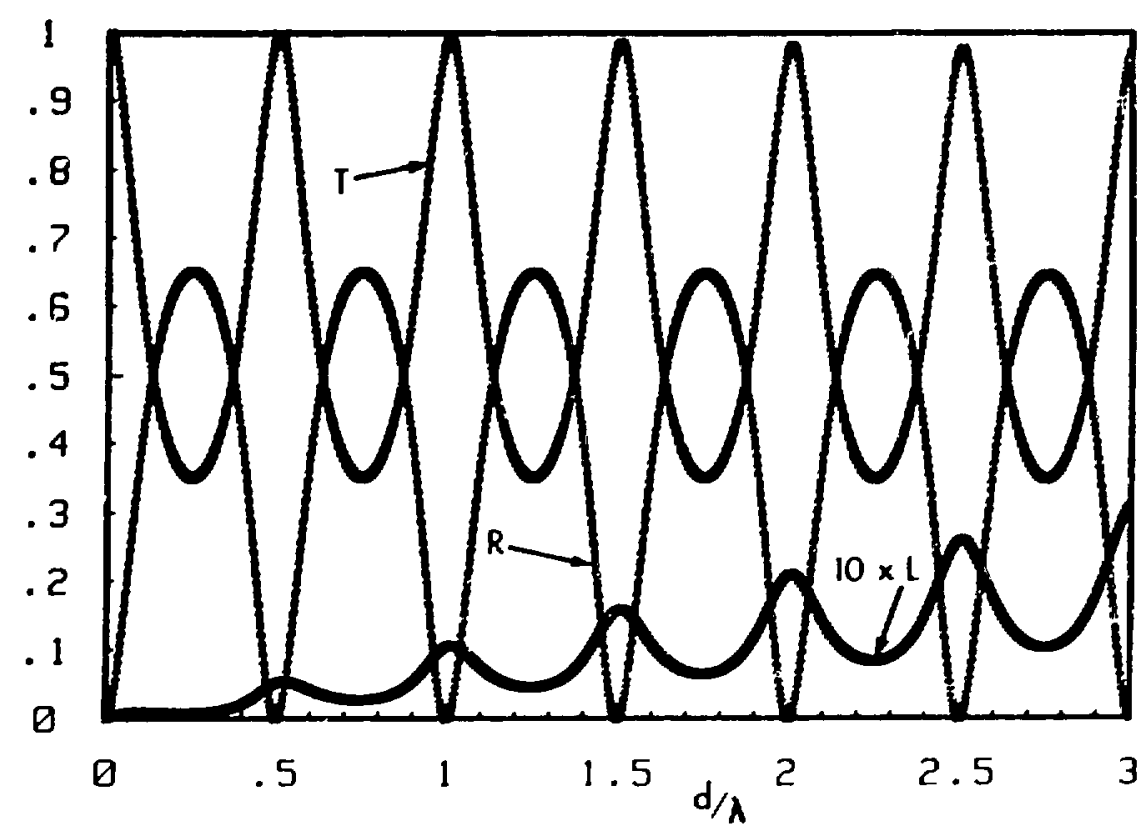

Fig. 14.

Reflection, transmission, and loss $\times 10$ for $\mathrm{Al}_{2} \mathrm{O}_{3}$ slab showing thickness dependence on wavelength in material. $=9.3$.

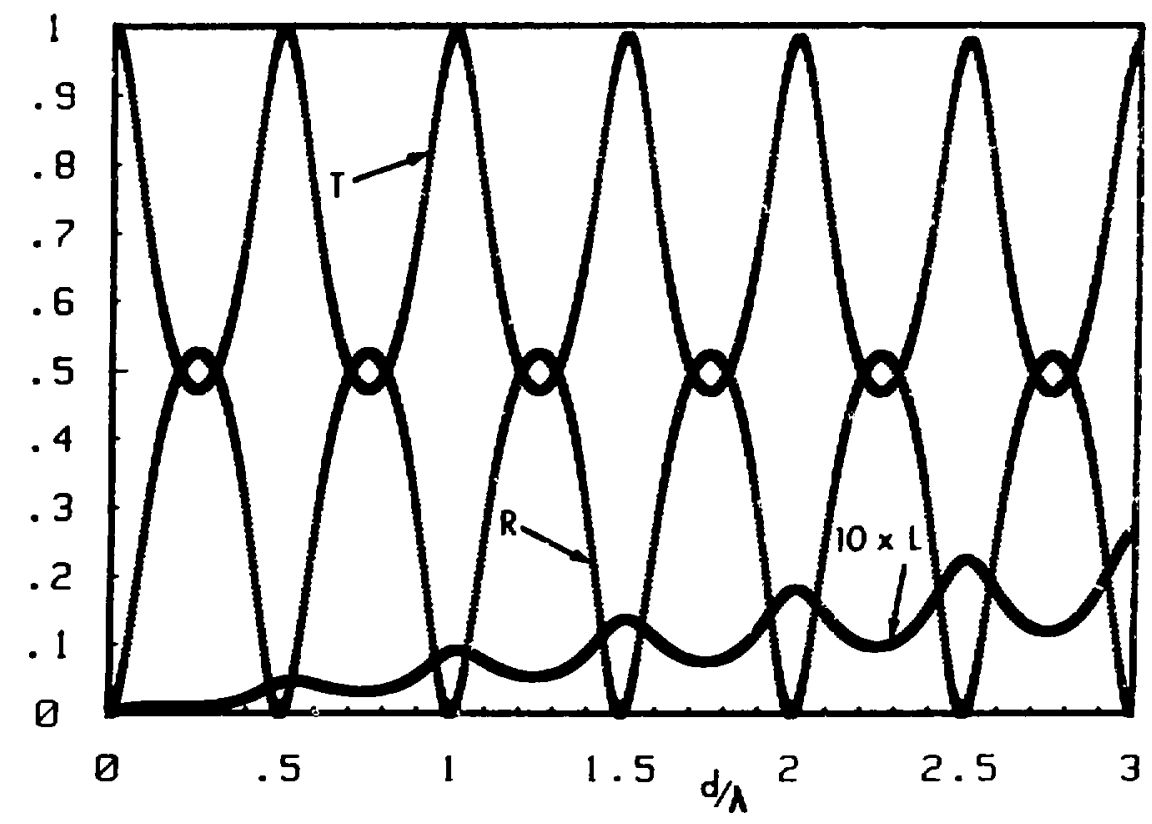

Fig. 15.

Same as Fig. 14 but for BeO $\left(\kappa^{\prime}=6.3\right)$. 


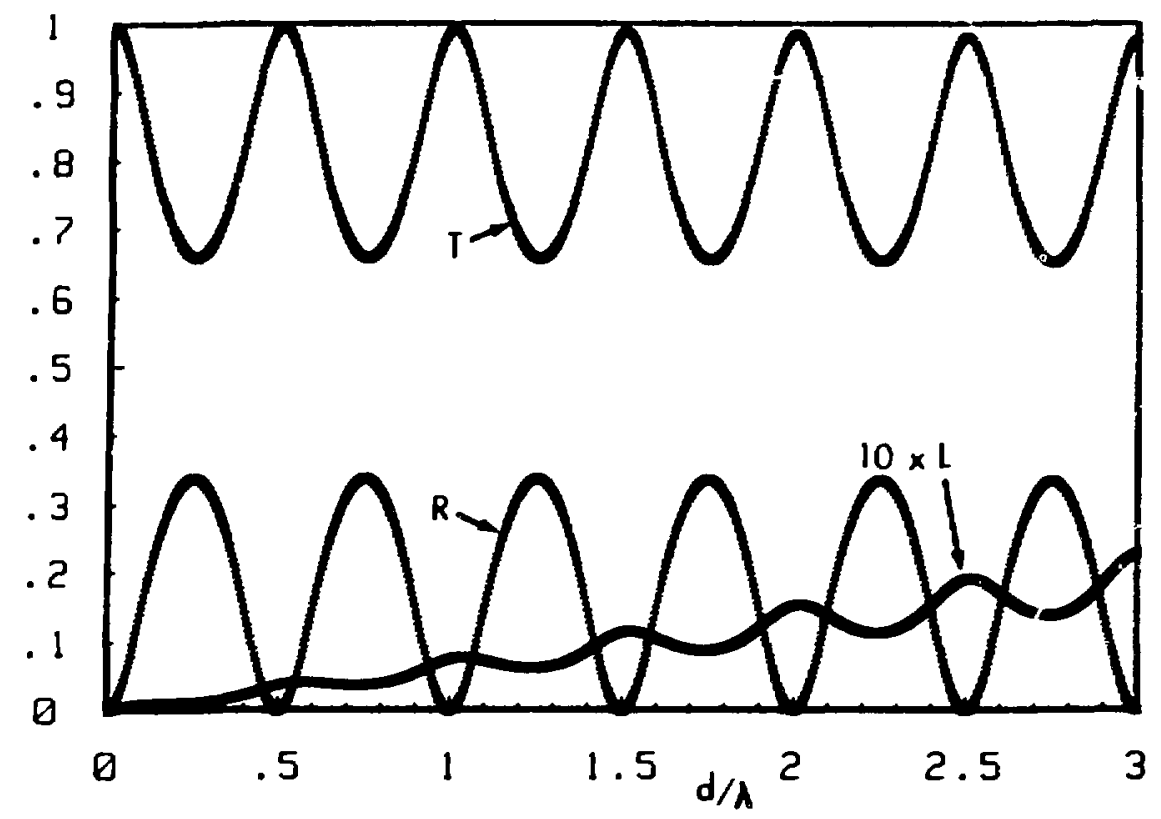

Fig. 16.

Same as Fig. 14 but for $\operatorname{SiO}_{2}\left(\kappa^{\prime}=3.8\right)$.

The wavelength in the materlal is given by Eq. (31). Just as for the phase factor $B, F i g$. $11 b$, the wavelength depends strongly on $k^{\prime}$ and only weakly on tan $\delta$. Relative wavelength is plotted as a function of relative dielectric constant in Fig. 17.

c. Calculation of Initial Rate of Temperature Rise

Given a slab at initial uniform temperature and heat input $A(x)$, the initial rate of increase in temperature can be calculated using Eq. (56) with no thermal gradients and thus no heat flow. In this case, Eq. (56) becomes

$\mathrm{dT} / \mathrm{dt}=\mathrm{A}(\mathrm{x}) / \rho \mathrm{c}$.

The heat inputs can be calculated from knowledge of the fields in the slab, Eq. (53c), using the Poynting vector, Eq. (50). A computer program was written to perform the necessary complex arithmetic. The output of this program is the heat introduced into the slab per unit area per unit length as a function of distance into the slab.

Results of calculations based on this program and using Eq. (69) are shown in Fig. 18 for alumina. The results for beryllia are almost the same, whereas 


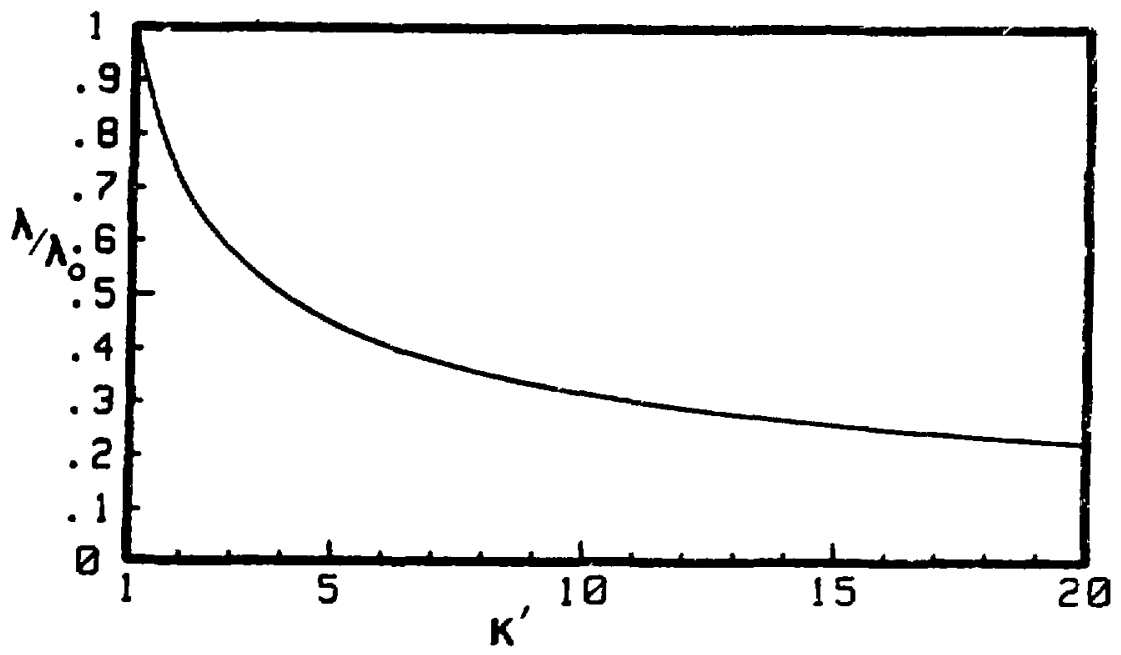

Fig. 17.

Wavelength in the material vs dielectric constant.

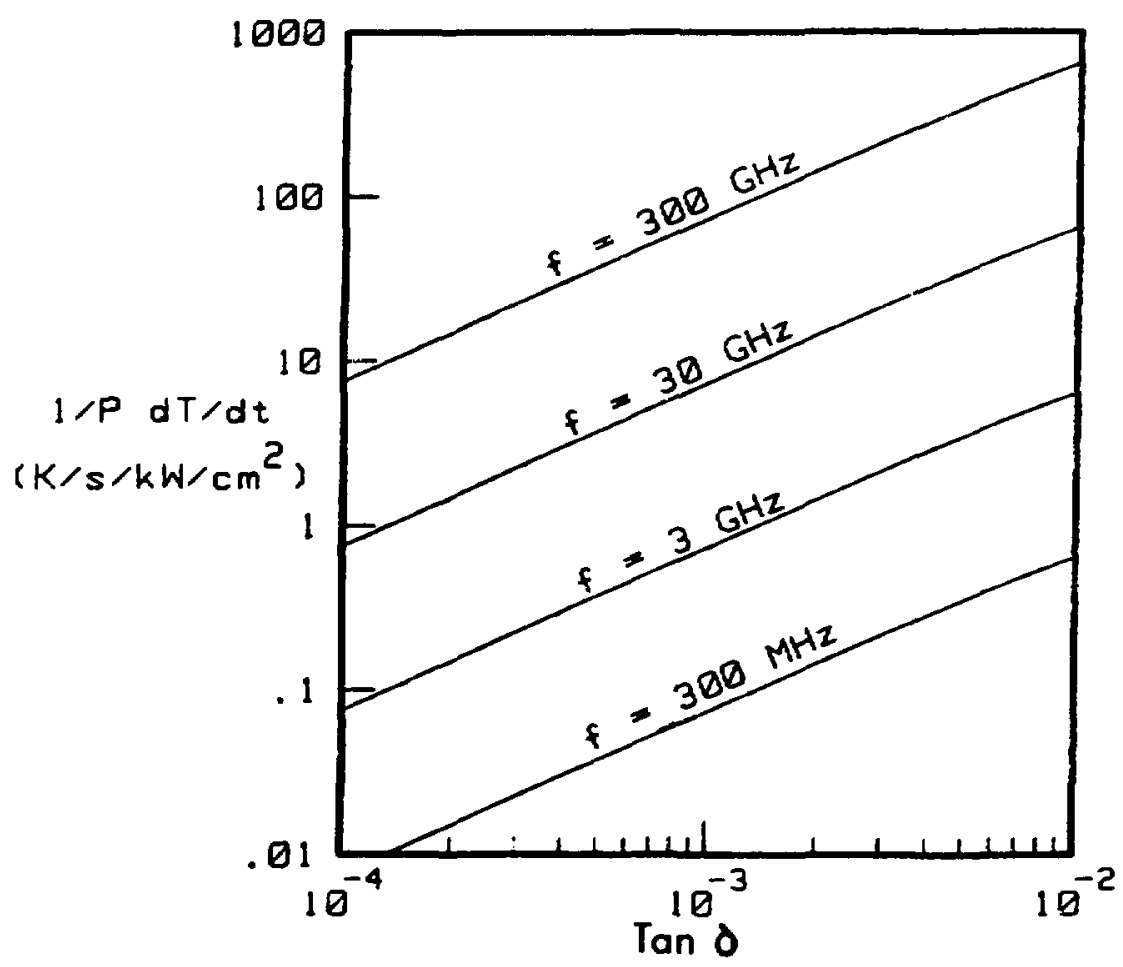

Fig. 18.

Relative rate of initial temperature rise per unit power input for a dielectric $\mathrm{slab}$ as a function of loss tangent for various frequencies. 
for fused sllica the lines would fall about $30 \%$ below these. The initial rate of temperature rise per $\mathrm{kW} / \mathrm{cm}^{2}$ input is averaged throughout the $s$ lab in this $f i g-$ ure. For slabs which are only a few integral multiples of a half wavelength thick (or less), the initlal rate of temperature rise is not strongly dependent on distance into the slab. In these calculations, the slab thicknesses were set to two times the wavelength in the material.

As can be seen from this figure, fnitlal iseating is a strong function of loss tangent and frequency. As these two parameters increase, the initial rate of temperature rise increases also. This dependerce on frequency implies that windows used at electron cyclotron resonance frequencies (Table I) will get much hotter than wisdows for ion cyclotron use, all other variables being equal. D. Calculated Temperature Profiles

Time-dependent temperature profiles in windows can be calculated by linking the R. F. loss computer program with the program which calculates heat flow by finite differences.

First a "reference case" will be presented, followed by results for sclected single-parameter departures. Values for the reference case are presented in Table II.

Window thicknesses, also shown in Table II, were selected to make them similar to each other. Thermal conductivities for $\mathrm{Al}_{2} \mathrm{O}_{3}$ (sapphire) and BeO were analytically modeled in temperature dependence from experimental data. 23 The thermal conductivity of fused silica is not strongly temperature dependent near room temperature, $^{23}$ so a constant value was used in the calculations.

Figures 19-21 show the results for the reference case at selected times.

TABLE II

\section{PARAMETER VALUES FOR REFERENCE CALCULATIONS}

Input power: $1 \mathrm{~kW} / \mathrm{cm}^{2}$. Frequency: $30 \mathrm{GHz}$. Number of zones: 6 . Loss tangent: 0.001. Cooling mode: One face at $300 \mathrm{~K}$, no heat flow across other face.

Materials

density $\left(\mathrm{g} / \mathrm{cm}^{3}\right)$

dielectric constant

thickness (mm)

heat capacity ( $\mathrm{cal} / \mathrm{g} \mathrm{K}$ )

thermal conductivity ${ }^{23}$ temperature

(cal $/ \mathrm{cm} s \mathrm{~K}$ )

$$
\mathrm{Al}_{2} \mathrm{O}_{3}
$$

9.3

$6.558(2 \quad \lambda)$

.26

dependent

$\begin{array}{rr}\mathrm{BeO} & \mathrm{SiO}_{2} \\ 3.01 & 2.60 \\ 6.3 & 3.8\end{array}$

$7.968(2 \lambda)$

.24

temperature

dependent $5.130(\lambda)$

.26

$3.34 \times 10^{-3}$ 


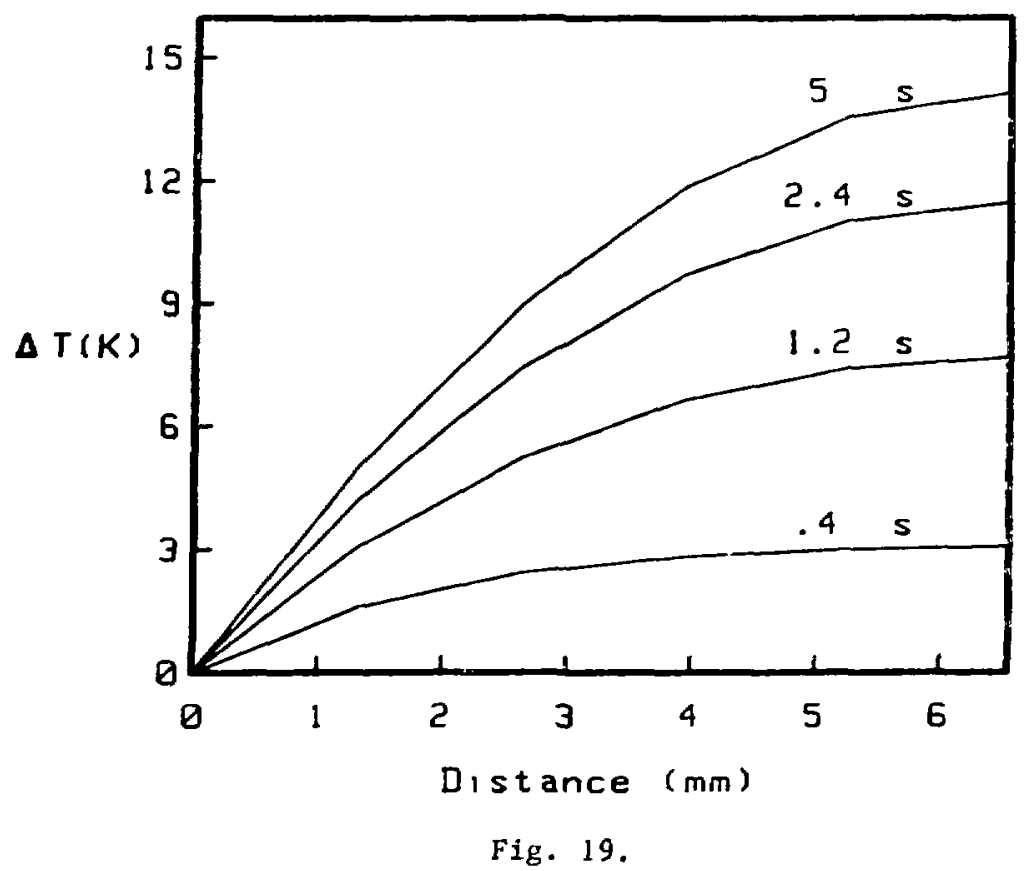

Calculated temperature distributions at selected times in a slab of $\mathrm{Al}_{2} \mathrm{O}_{3}$ for reference conditions (Table II). Base temperature is $300 \mathrm{k}$.

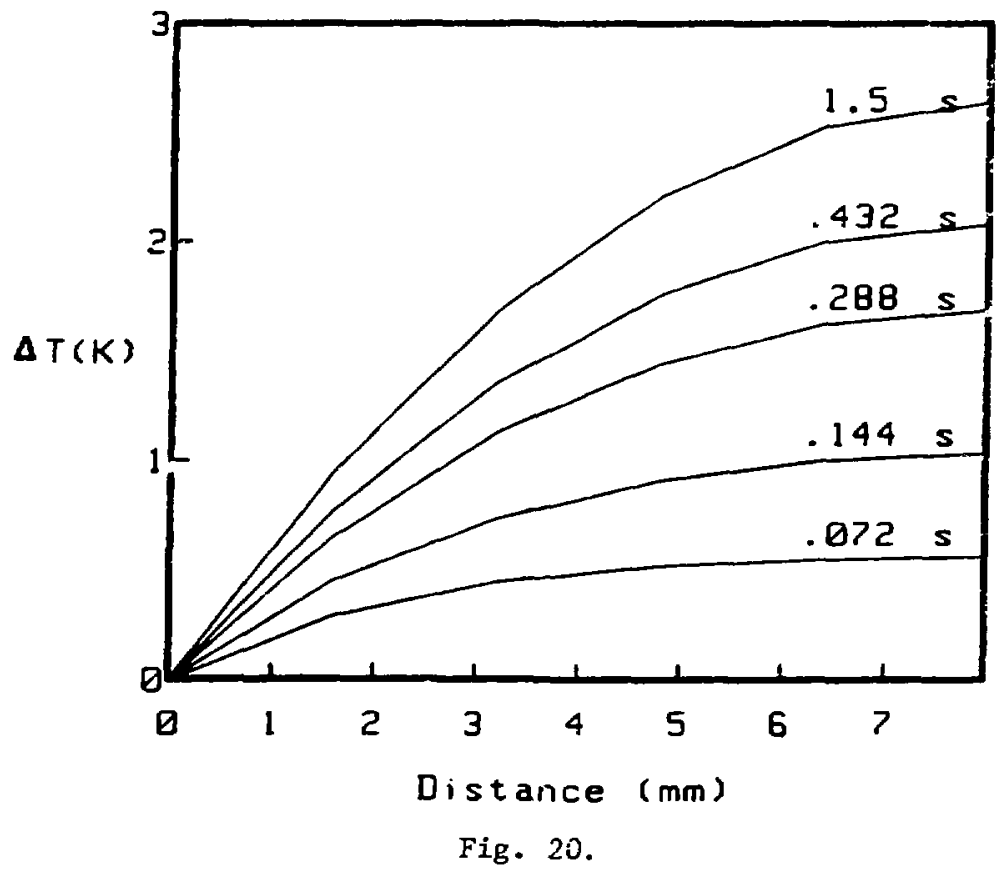

Calculated temperature rise above $300 \mathrm{~K}$ for a slab of BeO at selected times for reference conditions (Table II). 


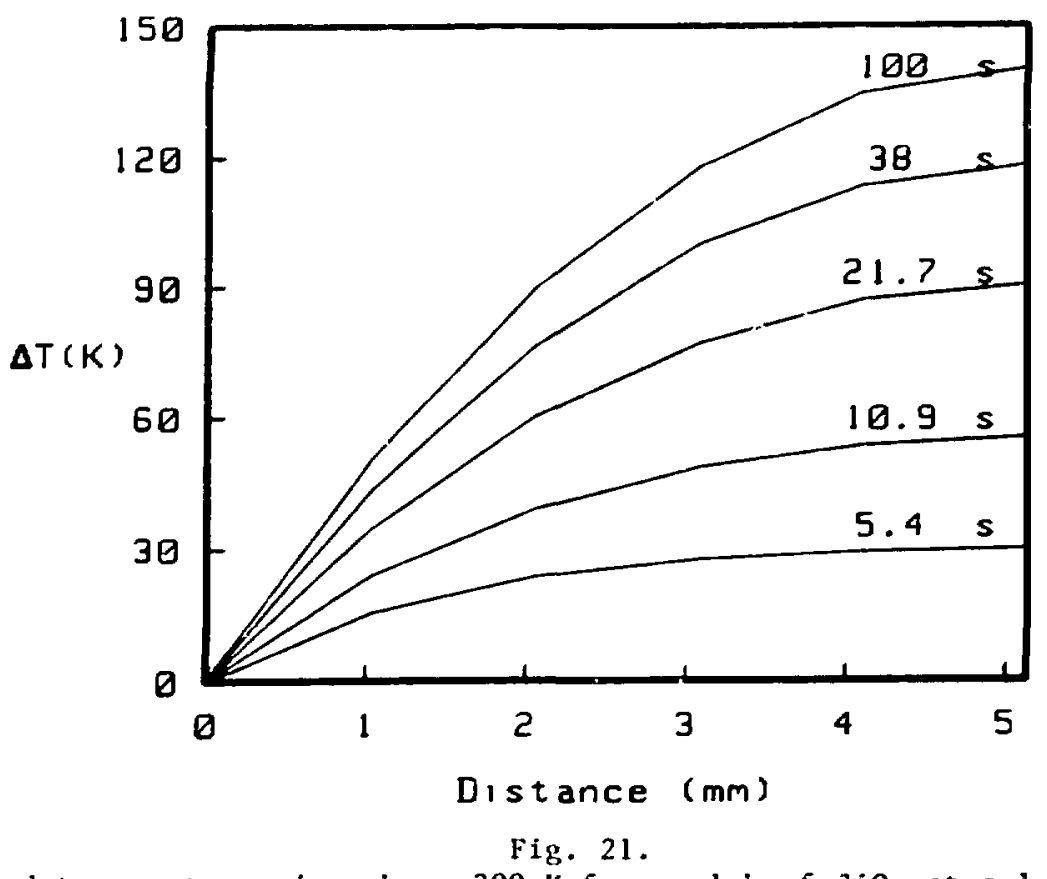

Calculated temperature rise above $300 \mathrm{~K}$ for a slab of $3 i 0_{2}$ at selected times for reference conditions (Table II). Thickness is one wavelength.

The wide range of final temperatures is due mostly to the large differences in thermal conductivities among the three materials. Beryllia has by far the largest thermal conductivity and fused silica the lowest.

Figure 22 shows time dependence of the uncooled faces for the three materials. Because of its smaller dielectric constant, fused silica starts to heat somewhat slower but at later times passes the other two materials. After $6 \mathrm{~s}$, which is the interval for startup heating (Table $I$ ), the temperature rise in $\mathrm{SiO}_{2}$ is about $40 \mathrm{~K}$ for $1 \mathrm{~kW} / \mathrm{cm}^{2}$ incident power.

1. Effects of Varying Thermal Conductivity. Using the initial temperature as a parameter departure from the reference case allows one to see the effects of the variable themal conductivities of $\mathrm{Al}_{2} \mathrm{O}_{3}$ and $\mathrm{BeO}$. Over the rango investigated, thermal conductivities for these tho materials increase substantially as temperature decreases. This implies that the temperature gradient across a cryogenically cooled window would be smaller than for the reference case. Calculations bear this out. Figure 23 shows the final (steady-state) temperature difference across slabs of $\mathrm{Al}_{2} \mathrm{O}_{3}$ and $\mathrm{BeO}$ as a function of initial temperature. All parameters except initial temperature can be found in Table II. It is apparent 


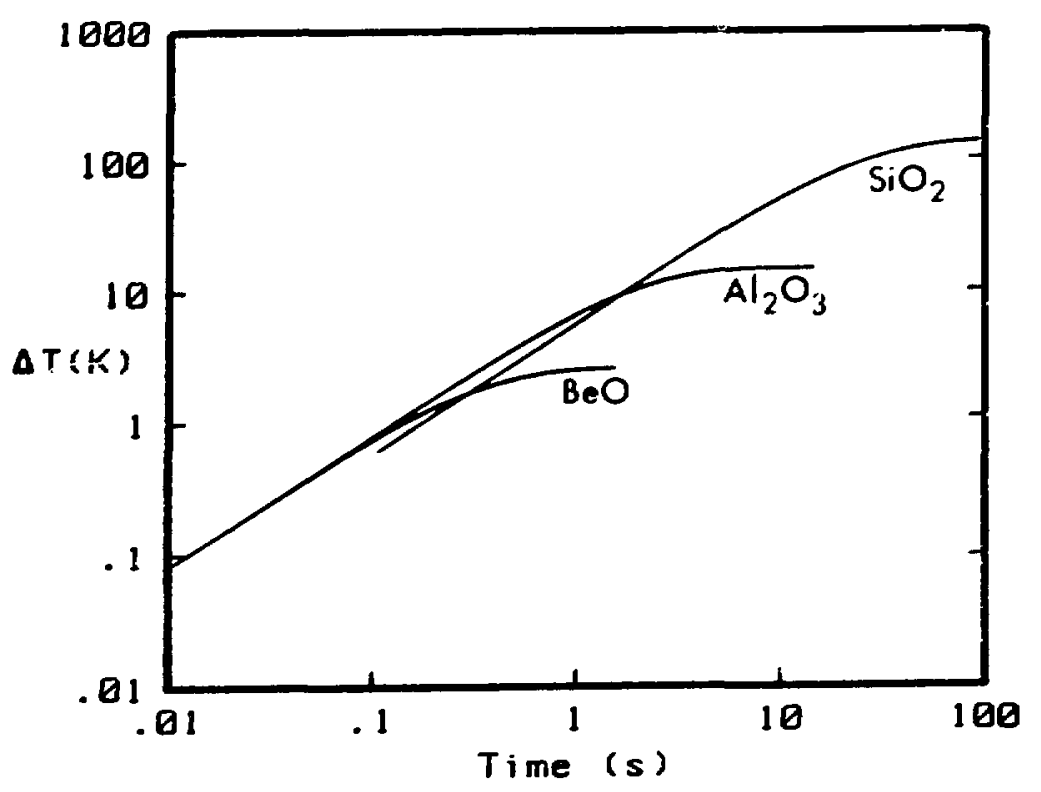

Fig. 22.

Temperature increases above $300 \mathrm{~K}$ for the rear (uncooled) faces of three naterials as a function of $t$ ime. Differences are due mainly to thermal conductivities.

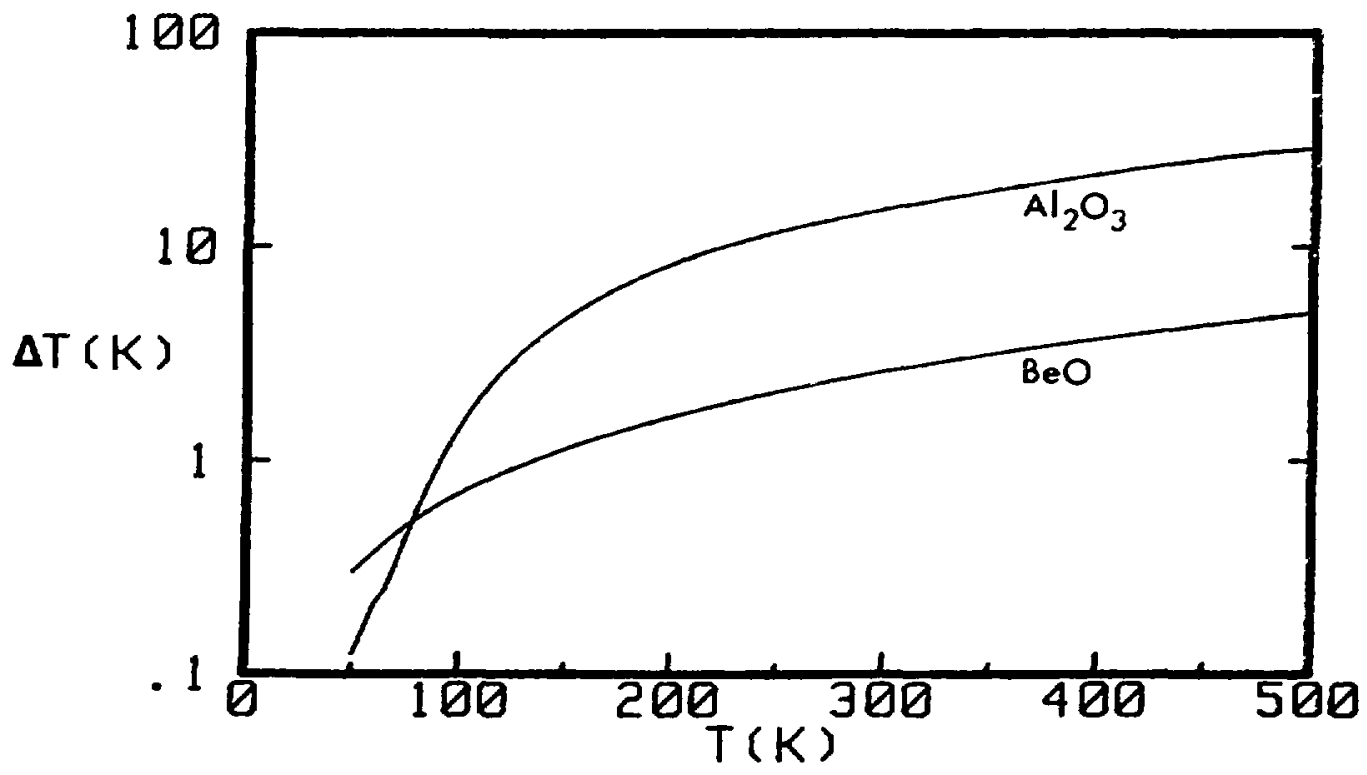

Fig. 23.

Final (steady-state) temperature gradient as a function of initial terperature for slabs of $\mathrm{Al}_{2} \mathrm{O}_{3}$ and $\mathrm{BeO}$. 
that a cooled window can work a factor of ten better (i. e. can handle 10 times the power input) than a room-temperature window. This does not hold true, however, for $\mathrm{SiO}_{2}$ because its thermal conductivity increases slightly with temperature. Thermal conductivity and its dependence on temperature is a very important consideration in selecting materials for R. F. windows.

2. Ef fects of Varying Loss Tangent. For sufficiently small values of the loss tangent, the attenuation factor is nearly proportional to it, as can be seen from Eq. (38). The attenuation factor enters inte the fields, Egs. (29) and (30), as a negative exponential which, for sinall values of the argunent, also scales proportionally. Thus it is expected that steady-state temperatures in a window will be almost directly proportional to loss tangent.

Calculated final temperature rises for a window of $\mathrm{Al}_{2} \mathrm{O}_{3}$ are plotted as a function of loss tangent in Fig. 24. Other parameters are as for the reference case (Table II). The line represents linear scaling from the lowest point. It is evident that over most of the range plotted, the expected proportionalit? occurs. Departures from this at the high end are due mostly to the decraising thermal conductivity of the material at high temperatures.

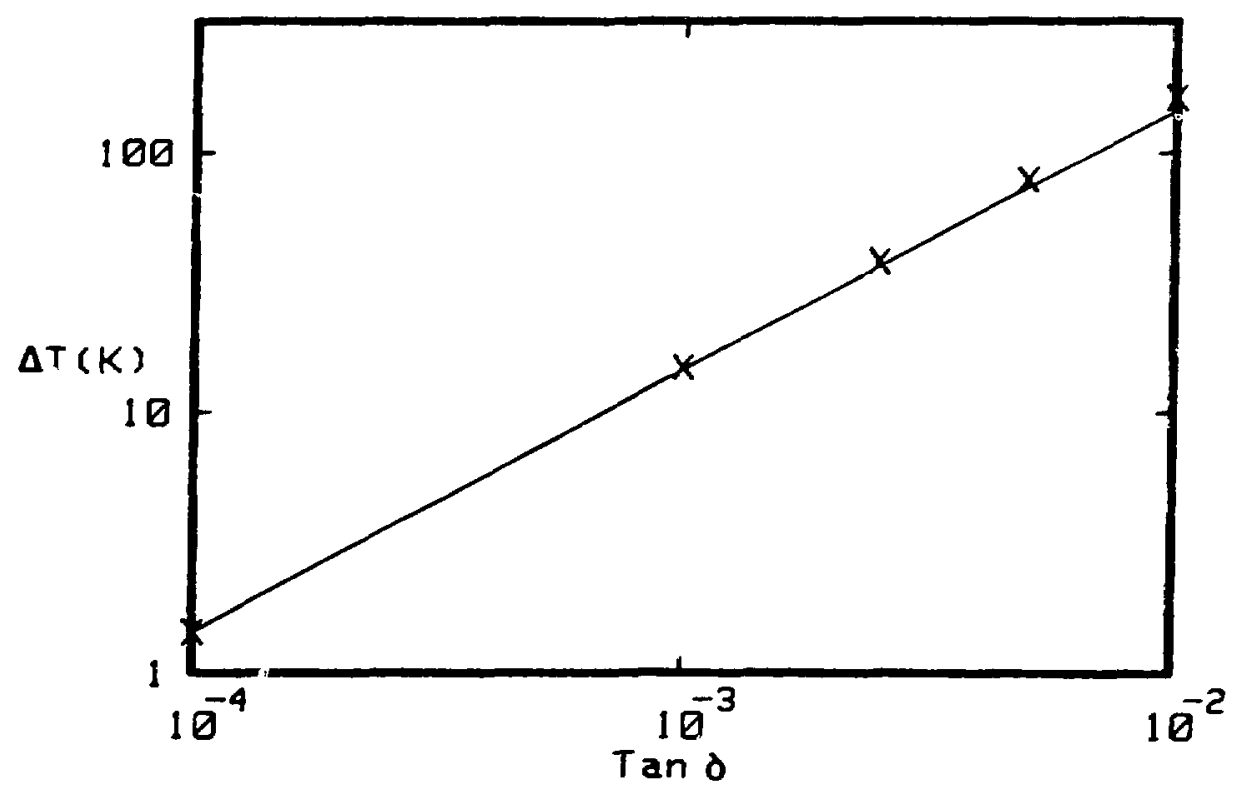

Fig. 24 .

Final temperature rises for a window of $\mathrm{Al}_{2} \mathrm{O}_{3}$ as a function of loss tangent. The line is a linear scaling from the lowest point. Points are calculated. 
3. Thickness Dependence. Using the same logic as in the previous section, but applying it to the distance into the material, rather than the loss tangent, it would appear that the heat deposited per unit volume is irdependent of thickness for sufficiently thin specimens. Once the heat is deposited, the flow is described approximately by Eq. (6I), where the material extends from zero to 1 . According to this equation, the final temperature distribution should be proportional to the square of the thickness, which is a parabolic distribution.

Calculations for an $\mathrm{Al}_{2} \mathrm{O}_{3}$ window with thickness varying from 0.5 wavel cngth to five wavelengths were undertaken to verify the quadratic nature of thre temperature dependence. A one wavelength thickness was $3.279 \mathrm{~mm}$. Results are shown in Fig. 25. The plotted points are the final temperatures on the uncooled face, ard the line is a quadratic scaling from the lowest point. It can be seen that, except at the highest temperatures, the proportionaity to the square is a good approximation. The depart:res at higher temperatures can be attributed to the decrease in thermal conductivity of $\mathrm{Al}_{2} \mathrm{O}_{3}$ with $\mathrm{rising}$ temperature.

As the thickness increases, the time required to reach equilibrium also pes up. This can be seen from Eq. $(60)$, where the terms inside the sumation represent the time dependence. As the thickness increases, it takes a longer time for

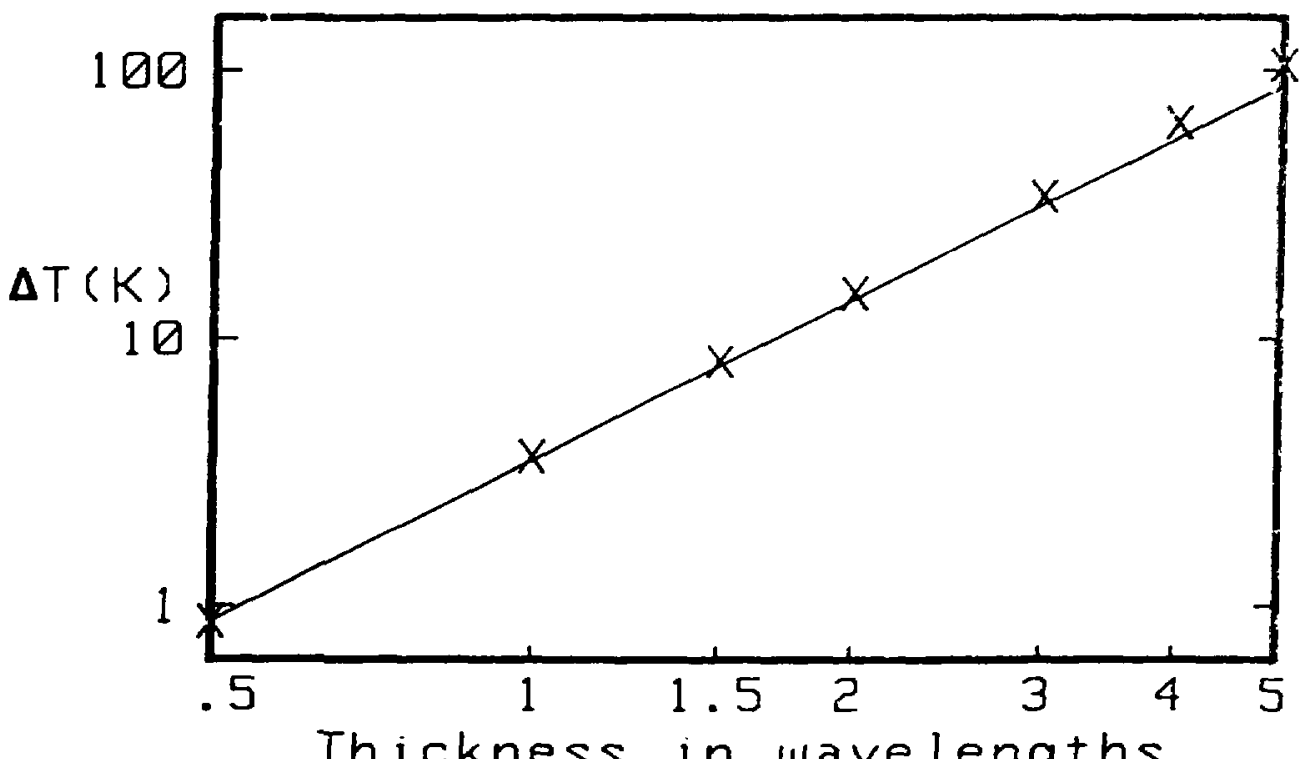

Thickness in wavelengths

Fig. 25.

Final temperature increases for $\mathrm{Al}_{2} \mathrm{O}_{3}$ as a function of thickness. The 1 ine is a quadratic extrapolation from the lowest point. 
the terms in the exponents to reach a given value. This dependence, as before, is on the square of the thickness, so the time should vary similarly. There is another factor which can change, however, and this is the thermal diffusivity $k=\mathrm{K} / \rho \mathrm{c}$. As the thermal conductivity decreases with heating, the diffusivity will also decrease, requiring slightly longer times than otherwise.

Times to reach 90 per cent of final temperature on the uncooled face are presented in Fig. 26 as a function of thickness. The line is a quadratic extension from the lowest point. It appears that this is a fair approximation to the calculated data.

4. Dependence on Input Power. Power levels in windows for fusion reactors may be much greater than the $1 \mathrm{kH} / \mathrm{cm}^{2}$ used in the reference calculation. To test the effect on final temperature of other power levels, a series of calculations was run with power inputs between 0.1 and $10 \mathrm{~kW} / \mathrm{cm}^{2}$. It would be expected that the variation of final temperacure would be proportional to the variation of power input, except for the already-nuted effect of decreasing thermal conductivity at high temperatures. This effect would make temperatures somewhat higher than otherwise expected.

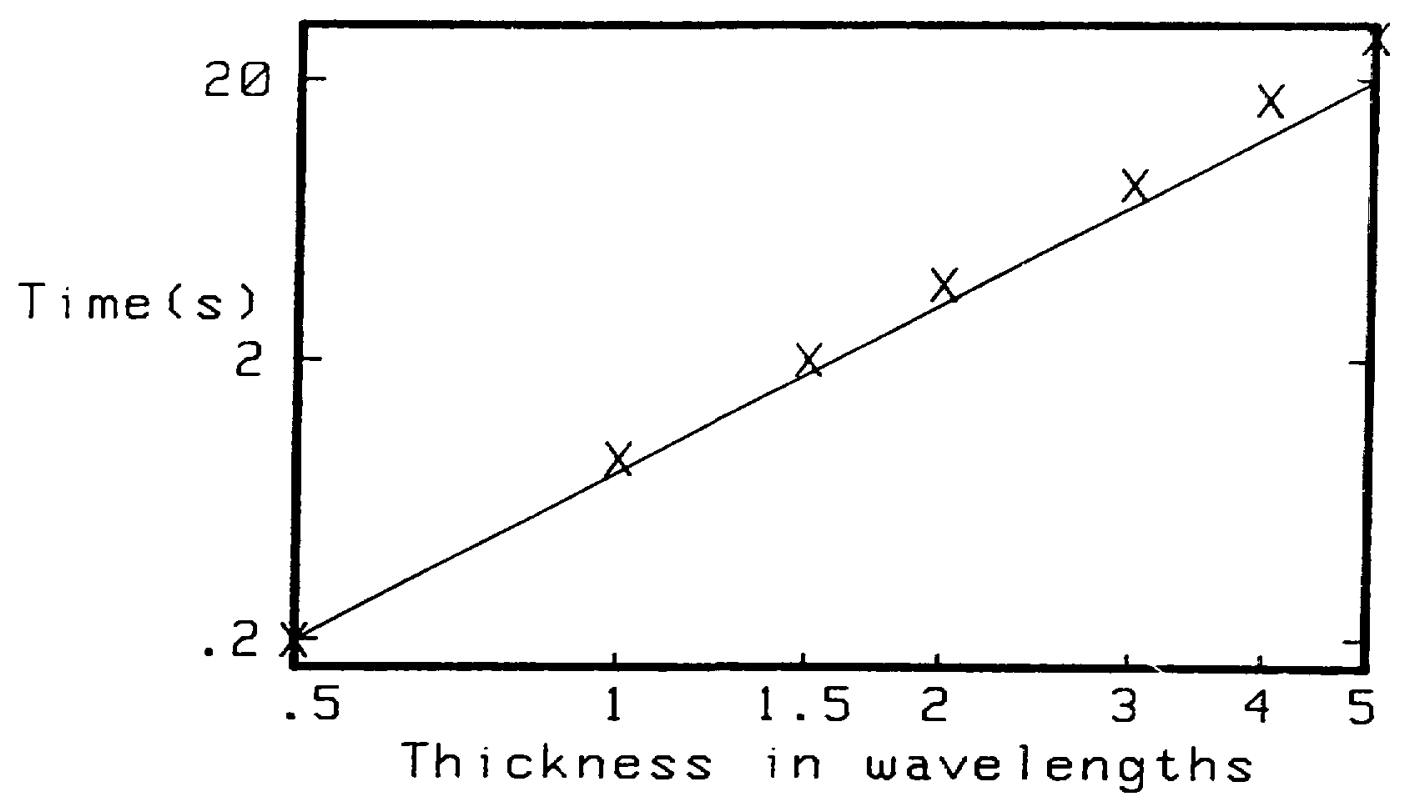

Fig. 26. Time to reach 90 per cent of final temperature on the unconled face for $\mathrm{Al}_{2} \mathrm{C}_{3}$.
Points are calculated and line is quadratic scaling from lowest point. 
Figure 27 shows a plot of final temperature increasc versus input power. The line is a linear dependence extrapolated from the lowest point. This appears to be a good approximation. At sufficiently high power levels, the final temperature is greater than would be expected from lintarity.

5. Effects of Frequency. As the frequency increases (wavelength decreases), onc would expect from Eq. (34) chat the attenuation factor and hence the loss would increase, with attendant temperature rise in the material. This meass that windows which might operate at a given frequency could fiall at higher frequencies, or, conversely, a material whlch wuld be unsuicable at 30 chiz would act acceptably at $30 \mathrm{MHz}$, which is in the ion cylotron heating range (Table 1 ). For example, $\mathrm{SiO}_{2}$, which gets quite hot at $30 \mathrm{CHz}$ (Fig. 21). would heal by anly C.007 $\mathrm{K}$ und $\mathrm{r}$ the same conditions at $50 \mathrm{MHz}$.

6. Future Calculations. It would be useful to extend the heat flow calculations to two dimensions so as to be able to calculate edge cooling effects in windows. To be able to simulate conditions in a waveguide with the electromagnetic equations would be useful also, as would be the provision for spatially and

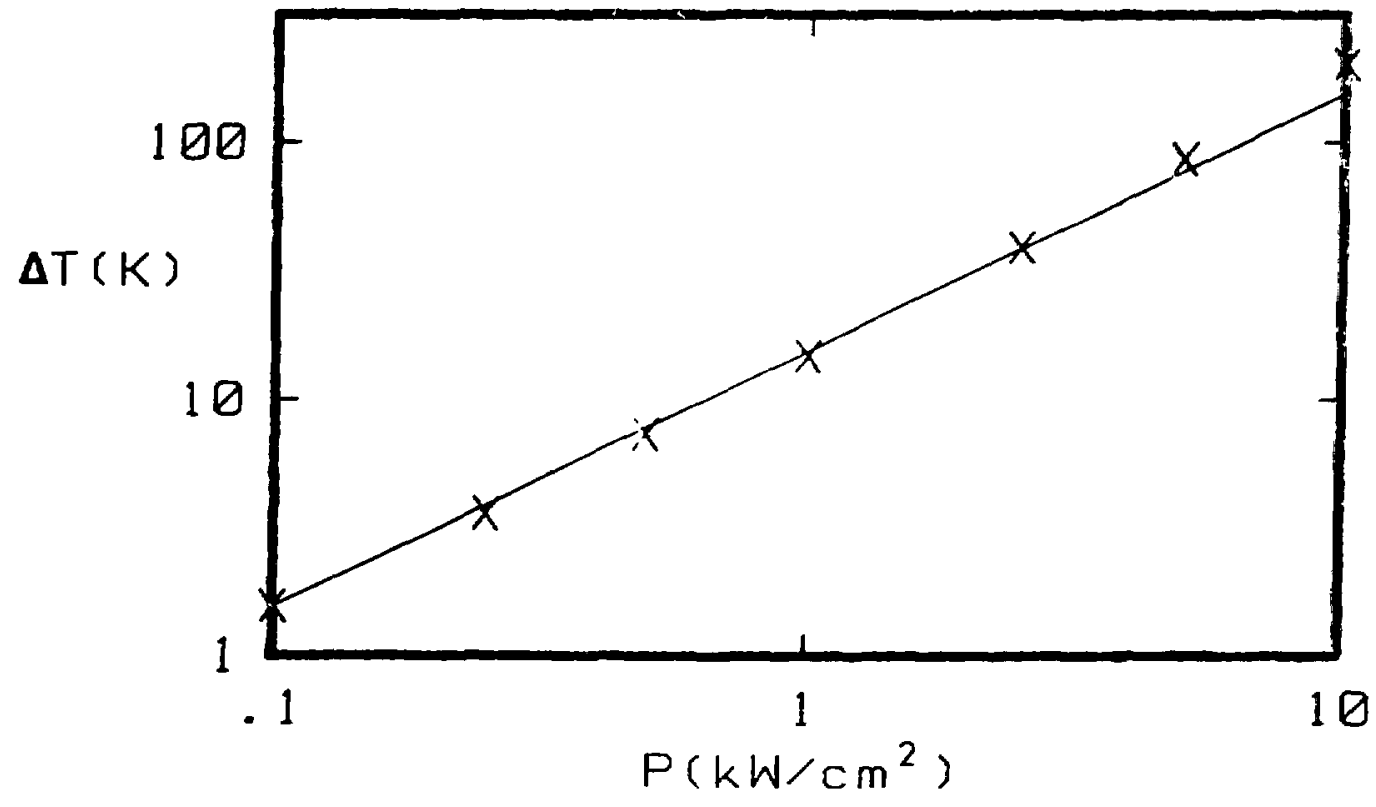

Fig. 27.

Temperature rise on the uncooled face of $\mathrm{Al}_{2} \mathrm{O}_{3}$ versus power input. Points are calculated; line is linear extrapolation from lowest point. 
temporally varying the loss tangent. This might require approximating solutions to the electromagnetic equations. Finally, since window failure occurs by cracking or fracture, these results would benefit by being coupled to a fracture analysis program.

v. SLMMARY

Operat ing environments for R. F. bindows in fusion reactors will be severe. Matcrials which pass all tests in the laboratory may act differently when placed in service, if actual operiting co'ditions are not simulated during the testing and selection phase. Although it is impossible currently to test window candidate naterials in the expected operating environment, every aitompt should be made to simulate conditions as realistically as possible.

Calculations provide useful information which can offer guidance in structuring line framework of an experimental program and can amplify and extrapolate results into areas which cannot presently be simulated adequately.

On the basis of calculations presented here, the following conclusionsi are reached.

1. The best impedance matches are obtained with windows which are multiples of a half wavelength thick.

2. There are large variations in final temperature distributions among candidate materials dut to variations in thermal conductivity.

3. Temperatures scale approximately proportionally with loss tangent and power input.

4. Heating rates are affected somewhat by changes in dielectric constant.

5. Temperatures and times to reach equilibrium scale approximately with the square of the thickness for face-cooled windows.

6. Temperatures scale approximately inversely with wavelength. Some materials which are unacceptable at high frequencies may find use at sufficiently low frequencies.

7. Calculations are relatively inexpensive and, it is hoped, can provide useful information to R. F. window designers.

\section{REFERENCES}

1. F. Johnson, "High Power R-F Window Study Program," Varian Associates report 304-1F (1963).

2. F. W. Clinard, Jr. and G. F. Hurley, "Ceramic and Organic Insulators for Fusion Applications," J. Nucl. Mat. (to be published). 
3. J. D. Walton, Jr., Radome Engineering Handbook (M. Dekker, Inc., New York, 1970).

4. Anon., "ETF Interim Design Document," ETF Design Center, Oak Ridge National Laboratory (1980).

5. C. A. Flanagan, D. Steiner, and G. E. Smizil, "Initial Trade and Design Studies for the Fusion Engineering Device," Oak Ridge National Laboratory report ORNL/TM-7777 (1981).

6. W. D. Kingery, H. K. Bowen, and D. R. Uhlmann, Introduction to Ceramics (John Wiley \& Sons, New York, 19;6), Pp. 913-964.

7. A. von Hippel, Dielectric Materlals and Applications (John Wiley \& Sons, New York, 1954), pp. 3-46.

8. J. A. Stratton, Electromagnet ic Theory (McGraw-Hil1, New York, 1941), pp. 511-515.

9. A. von Hippel, Dielectrics and Waves (M. I. T. Press, Cambridge, 1954).

10. R. E. Collin, Field Theory of Guided Waves (McGraw-Hill, New York, 1960).

11. H. S. Carslaw and J. C. Jaeger, Conduction of Heat in Solids (Clarendon Press, Oxford, 1978).

12. G. M. Dusinberre, Heat-Transfer Calculations by Finite Differences (International Textbook Co., Scranton, Pa., 1961).

13. J. A. Stratton, op. cit., p. 516.

14. H. Jory, S. Evans, S. Hegji, and J. Shively, "Development Program for a $200 \mathrm{~kW}, \mathrm{CW}, 28-\mathrm{GHz}$ Gyroklystron," Oak Ridge National Laboratory report ORNL/Sub/01617/8 (1978).

15. J. F. Shively, T. J. Grant, A. L. Nordquist, D. S. Stone, and G. E. Wendel1, "60 GHz Gyrotron Development Program," Oak Ridge National Laboratory report ORNL/Sub-79/21453/5 (1980).

16. J. A. Topping and J. O. Isard, "The Dielectric Properties of Sodium Aluminosilicate Glasses at Microwave Frequencies," Phys. Chem. Glasses 12 , 145-151 (1971).

17. W. George and P. Popper, "The Dielectric Properties of Some Commercial Alumina Materials at $9368 \mathrm{MHz}$," Proc. Brit. Ceram. Soc. 10, 63-78 (1978).

18. W. George, "Electrica? Properties of Some Commercial Beryllia Ceramics," Proc. Br. Ceran. Soc. 72, 62-71 (1972).

19. M. N. Afsar and K. J. Button, "Millimeter and Submillimeter Wave Measurements of Complex Optical and Dielectric Parameters of Materials," (to be published).

20. J. B. MacChesney ard G. E. Johnson, "Room-Temperature Dielectric Properties of Fast-Neutron-Irradiated Fused Silica and alpha Alumina," J. Appl. Phys. 35, 2784-2785 (1964).

21. P. P. Budnikov, F. G. Kerbe, and N. S. Kostyukov, "Influence of Irradiation by Thermal Neucrons on Certain Electrical Properties of Ceramics Made from Pure Aluminum Oxide," Izvest. Akad. Nauk USSR, Neorgan. Mater. 3, 94-100 (1967).

22. R. W. Klaffky, B. H. Rose, A. N. Goland, and G. J. Dienes, 
"Radiation-Induced Conductivity of $\mathrm{Al}_{2} \mathrm{O}_{3}$ : Experiment and Theory," Phys. Rev. B 21, 3610-3634 (1980).

23. Y. S. Touloukian, R. W. Powe11, C. Y. Ho, and P. G. Klemens, Thermal Conductivity (Plenum, New York, 1970). 